\title{
Spectral-Domain Optical Coherence Tomography in Hereditary Retinal Dystrophies
}

\author{
Isabelle Meunier et al. ${ }^{*}$ \\ Centre national de Référence Maladies Sensorielles Génétiques, Hôpital Gui de Chauliac \\ and INSERM U583, Institute for Neurosciences of Montpellier,
}

France

\section{Introduction}

Inherited retinal diseases are rare disorders that affect 40000 to 50000 individuals in France. They represent $20 \%$ of cases of blindness in subjects younger than 20 years of age. There is great clinical and genetic diversity among these dystrophies: diversity at the clinical level ranging from mild maculopathies to Leber's congenital amaurosis; diversity among genes and modes of transmission (more than 150 known genes expressed in the retina). Nonsyndromic retinitis pigmentosa is the most common among hereditary dystrophies (fig 1) (Berger et al, 2010).

Devices for time-domain optical coherence tomography and, more recently, spectral-domain optical coherence tomography (spectral OCT) with an improved depth resolution of $7 \mu \mathrm{m}$ (wavelength 840nm, scannong speed 27000 to 40000 scans per second) carry out a noninvasive and in vivo visualization of the retinal layers, particularly the outer nuclear layer (ONL), the photoreceptor inner segment/outer segment junction (IS/OS) and the retinal pigment epithelium (RPE) (fig 2). Hence, the integrity or abnormality of the retinal pigment epithelium, the inner/outer segment junction, and the external limiting membrane can be analyzed even during early stages of inherited retinal diseases, when the fundus appears normal on examination.

Several commercial spectral OCT are available for posterior segment imaging (Cirrus HDOCT, Carl Zeiss Meditec, with a speed of 27000 scans per second and a resolution of $5 \mu$ Spectralis OCT, Heidelberg Engineering, with a speed of 40000 scans per second and a resolution of $7 \mu$ - RTVue-100, Optovue, with a speed of 26000 scans per second and a

\footnotetext{
${ }^{*}$ Carl Arndt ${ }^{2}$, Xavier Zanlonghi ${ }^{3}$, Sabine Defoort-Dhellemmes ${ }^{4}$, Isabelle Drumare ${ }^{4}$

Martine Mauget-Faysse ${ }^{5}$, Benjamin Wolff ${ }^{5}$, Aude Affortit ${ }^{6}$, Christian Hamel $^{1}$ and Bernard Puech ${ }^{4}$

1 Centre national de Référence Maladies Sensorielles Génétiques, Hôpital Gui de Chauliac and INSERM U583,

Institute for Neurosciences of Montpellier, France

2 Eye clinic, Hôpital Robert Debré, CHRU de Reims, France

3 Sourdille Clinic, Nantes, France

4 Service d'Exploration de la Vision et Neuro-ophtalmologie, Hôpital Robert Salengro, CHU de Lille, France

5 Centre d'Imagerie et de Laser Rabelais, Lyon, France

6 Rothschild Fondation, Pediatric Eye department, Paris, France
} 
resolution of $5 \mu$ - Copernicus HR, Optopol technologies, with a speed of 52000 scans per second and a resolution of $3 \mu$ ). OCT scans presented in this article were obtained with a Spectralis OCT, Heidelberg Engineering.

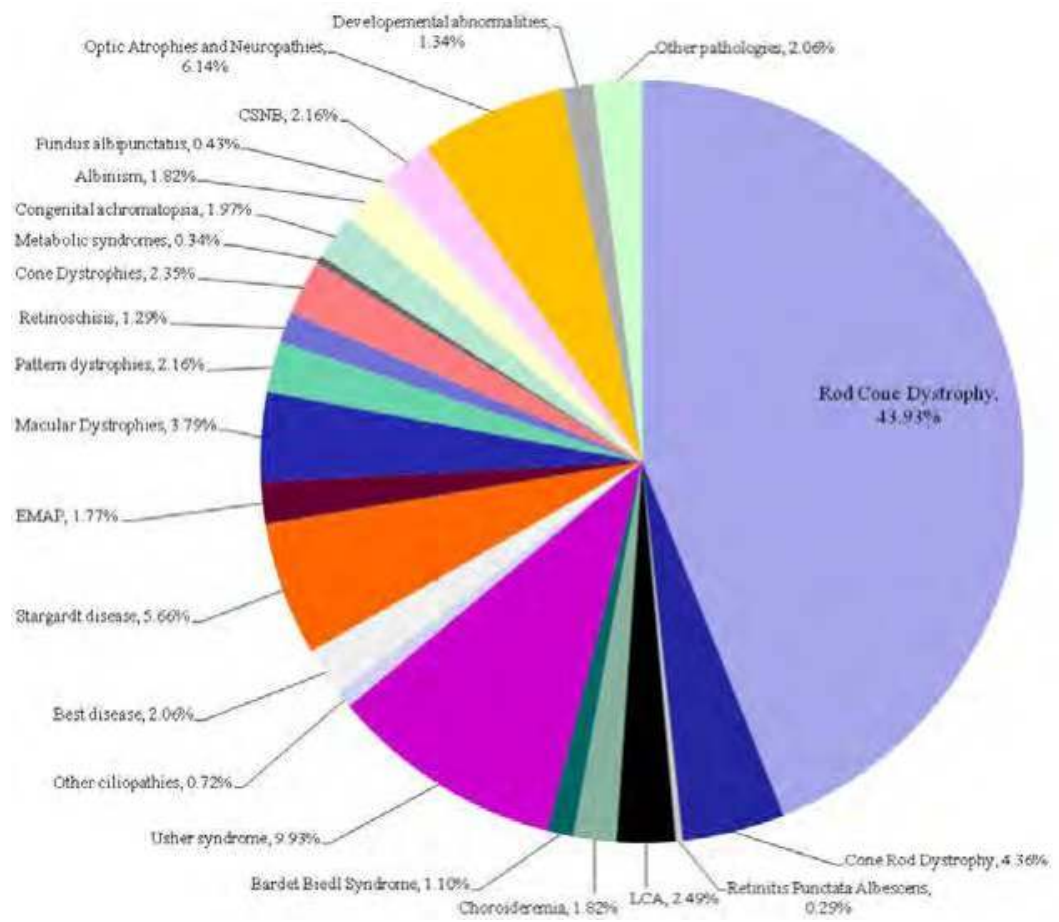

Fig. 1. Prevalence of retinal dystrophies by disease in terms of percentage of affected individuals, from the database of the National Center for Rare Sensory and Genetic Diseases, Montpellier, France. EMAP : Extensive macular atrophy with pseudo-drusen.

After this recapitulation of hereditary dystrophies and normal OCT scan, we will describe OCT anomalies in rod-cone (retinitis pigmentosa, RP), cone-rod (CRD) and progressive cone dystrophies (COD), followed by the principal hereditary maculopathies.

\section{Definition and classifications of hereditary retinal dystrophies}

A retinal dystrophy is to be considered when there is bilateral, symmetric (except in vitelliform dystrophies and pattern dystrophies) and evolutive visual damage in the absence of inflammatory, toxic or paraneoplastic causes. The evolutive character of the damage is an essential criterion related to the progressive degeneration of photoreceptors, which allows these disorders to be distinguished from other non-evolutive causes of loss of sight such as albinism, congenital essential night blindness and other congenital disorders (embryopathies). The notion of a familial disorder is an important argument, but its absence does not exclude a genetic cause (unique single cases of a recessive disorder). 

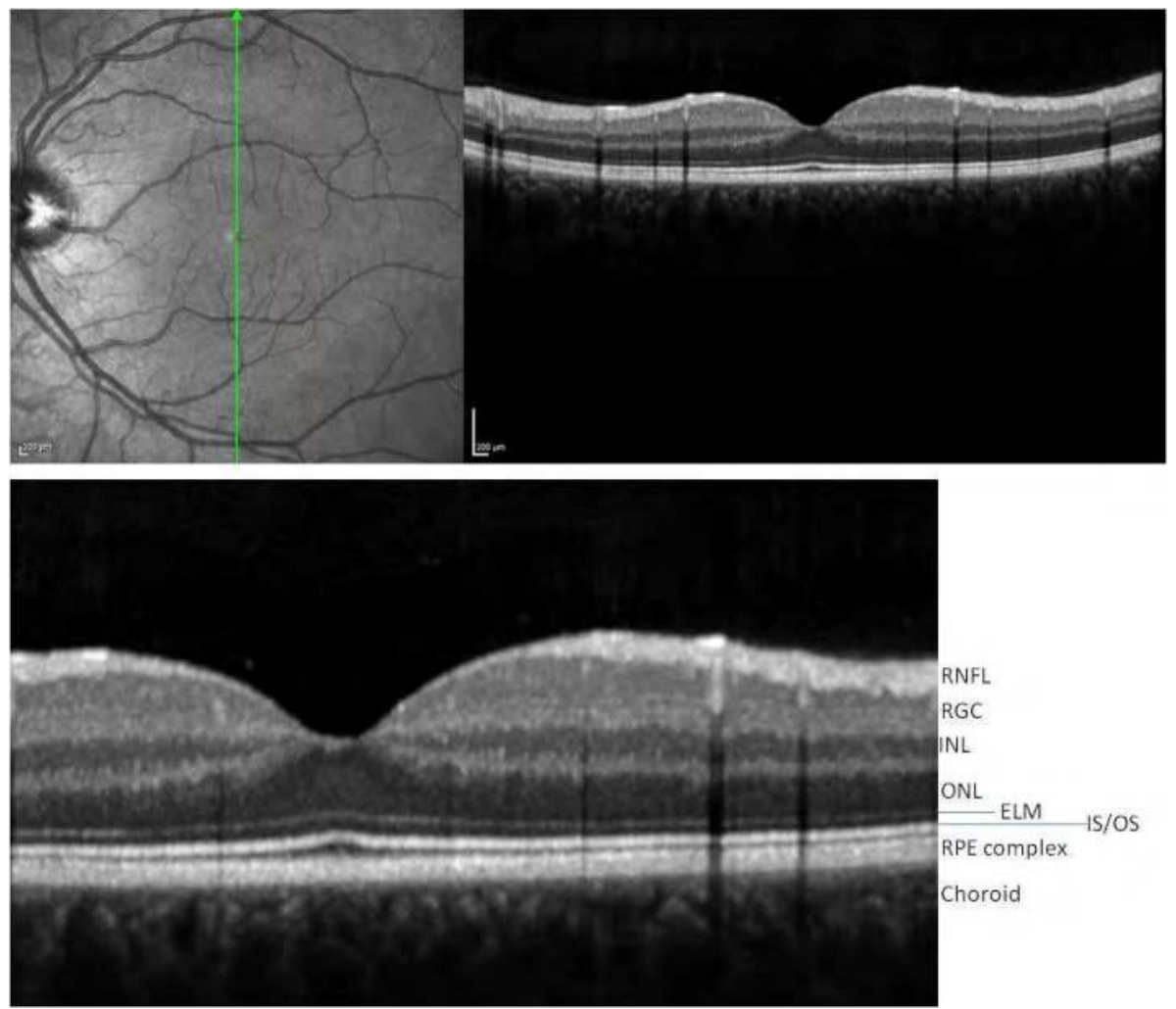

Fig. 2. Red free photography and corresponding OCT scan (top left and right) of a normal eye, in vivo visualization of the main retinal layers. Magnified OCT scan (bottom). RNFL: retinal nerve fiber layer. RGC: retinal ganglion cell layer, nuclei of the third set of neurons. INL: inner nuclear layer, nuclei of the second set of neurons. ONL: outer nuclear layer, nuclei of the first set of neurons, i.e. the photoreceptors. ELM: external limiting membrane. IS/OS: inner segment/outer segment. RPE: retinal pigment epithelium.

Macular diseases with or without autofluorescent deposits can be differentiated into progressive cone dystrophies, cone-rod dystrophies (cone-dominant diseases), retinitis pigmentosa (rod-dominant diseases), chorioretinal dystrophies and erosive vitroretinopathies. Macular diseases compromise central vision (loss of reading, writing and facial recognition functions), while mixed central and peripheral dystrophies can lead to a complete loss of vision.

From a genetic point of view, cones, rods or retinal pigment epithelial cells express the genetic dysfunction, but the damage may occur predominantly in the macular area due to differences in the level of metabolism or the deleterious accumulation of lipofuscin. In addition, a given gene could lead to different clinical profiles (macular damage, cone-rod dystrophy or rod-cone dystrophy) of varying severity, with inter- and intra-familial variations. For example, the genes involved in COD are also associated with CRD. 


\section{Spectral OCT in retinitis pigmentosa, cone-rod dystrophy and cone dystrophy}

In these dystrophies related to photoreceptor and RPE apoptosis, OCT in combination with fundus autofluorescence imaging is of great use in the analysis of the pattern and extent of neurosensory retinal disorganization in the macular zone. These modifications are apparent right from the early stages of the disease, before any macular changes are visible on fundus examination.

\subsection{Retinitis pigmentosa}

Retinitis pigmentosa is characterized by night blindness, primary progressive visual field defects and, eventually, the loss of central vision and legal blindness.

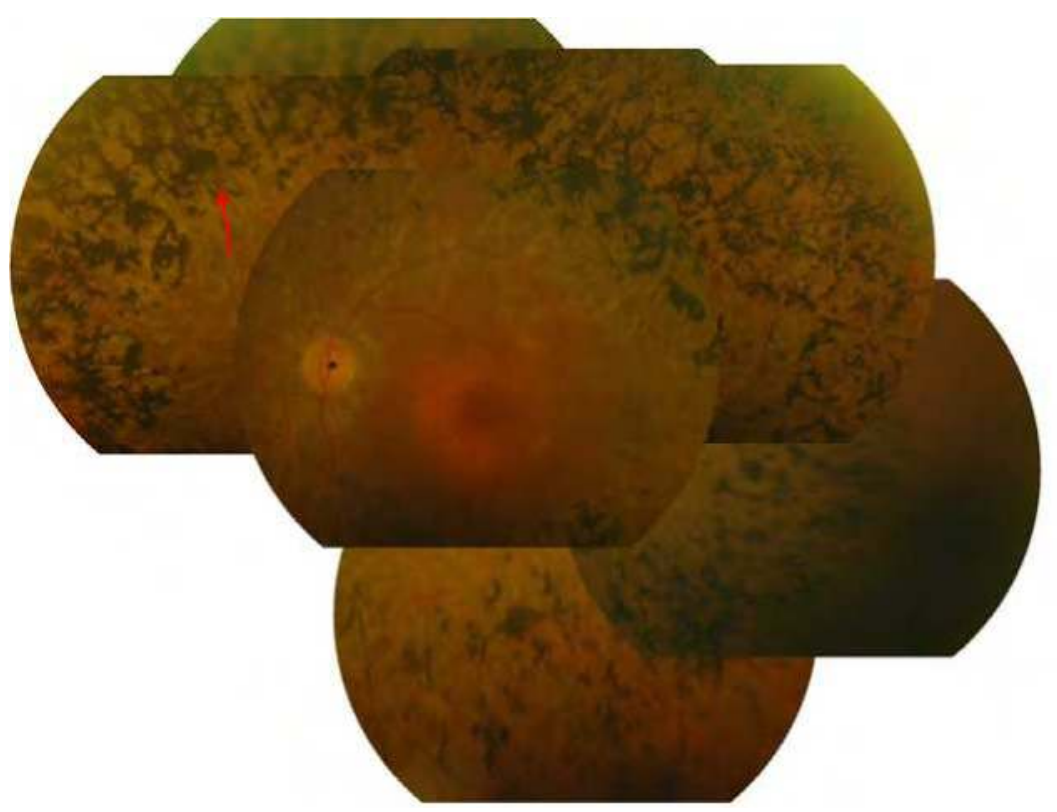

Fig. 3. A 32-year-old man with retinitis pigmentosa. Typical bony spicules of the peripheral retina (arrow), attenuation of retinal blood vessels. There is no pallor of the optic nerve head yet $\left(^{*}\right)$.

OCT reveals the disruption or disappearance of the inner segment/outer segment junction (IS/OS) at one or more extrafoveal locations from early stages, even though the macula is normal on fundus examination. Disorganization of the IS/OS junction is an early indication of dysfunction and loss of photoreceptors in retinitis pigmentosa. Progressive PR loss is subsequently reflected by a diminution of the thickness of the ONL (nuclei of photoreceptors) and RPE complex (fig 4-6). 


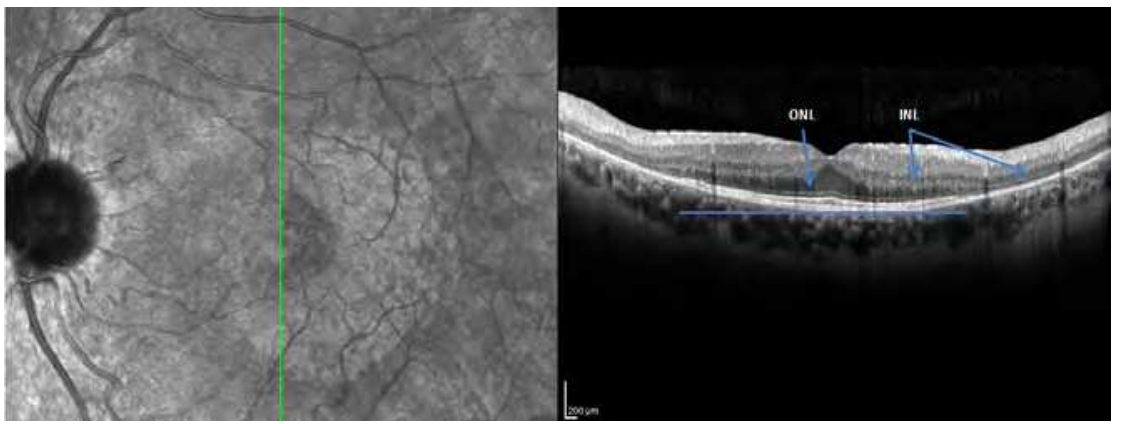

Fig. 4. Red free photography (left) and corresponding OCT scan of a 34-year-old man with retinitis pigmentosa. The IS/OS junction is only visible in the foveal zone, marked by a blue bar. Outside this zone, no IS/OS junction, ELM, or ONL is observed.

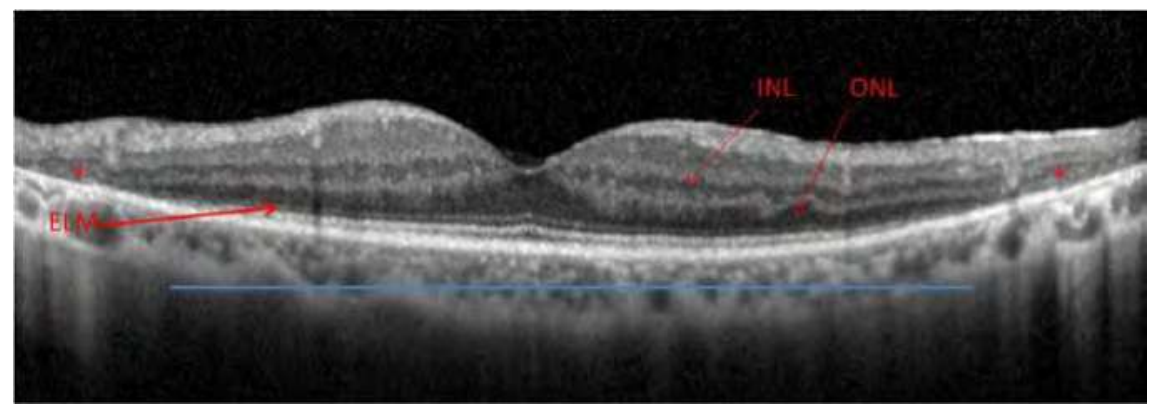

Fig. 5. OCT scan of a 20-year-old woman with retinitis pigmentosa. The IS/OS junction is visible in the entire foveal zone, marked by a blue bar. Outside the fovea, no IS/OS junction, ELM, or ONL is observed, and there is in addition a disorganization of the INL (*) with a marked reduction in total retinal thickness.

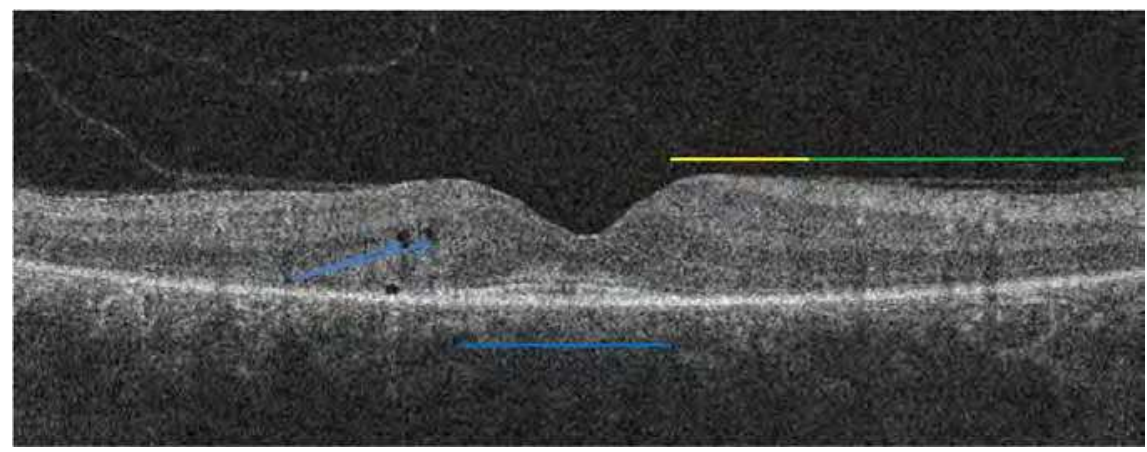

Fig. 6. OCT scan of a 32-year-old man with retinitis pigmentosa. Only the subfoveal part of the IS/OS junction is preserved (blue bar). Visual acuity is normal as long as the subfoveal IS/OS is present. Outside the macula, no IS/OS junction or ELM is observed (green bar). The ELM can still be noted in the perifoveal zone (yellow bar). Note several cystic spaces in the inner nuclear layer (blue arrows). 


\subsection{Progressive cone dystrophies}

Affected individuals report decreased visual acuity. They mention progressive intense photophobia and variable degrees of color vision abnormalities. The degenerative process predominates in cones but not in rods, as in retinitis pigmentosa (fig 6).

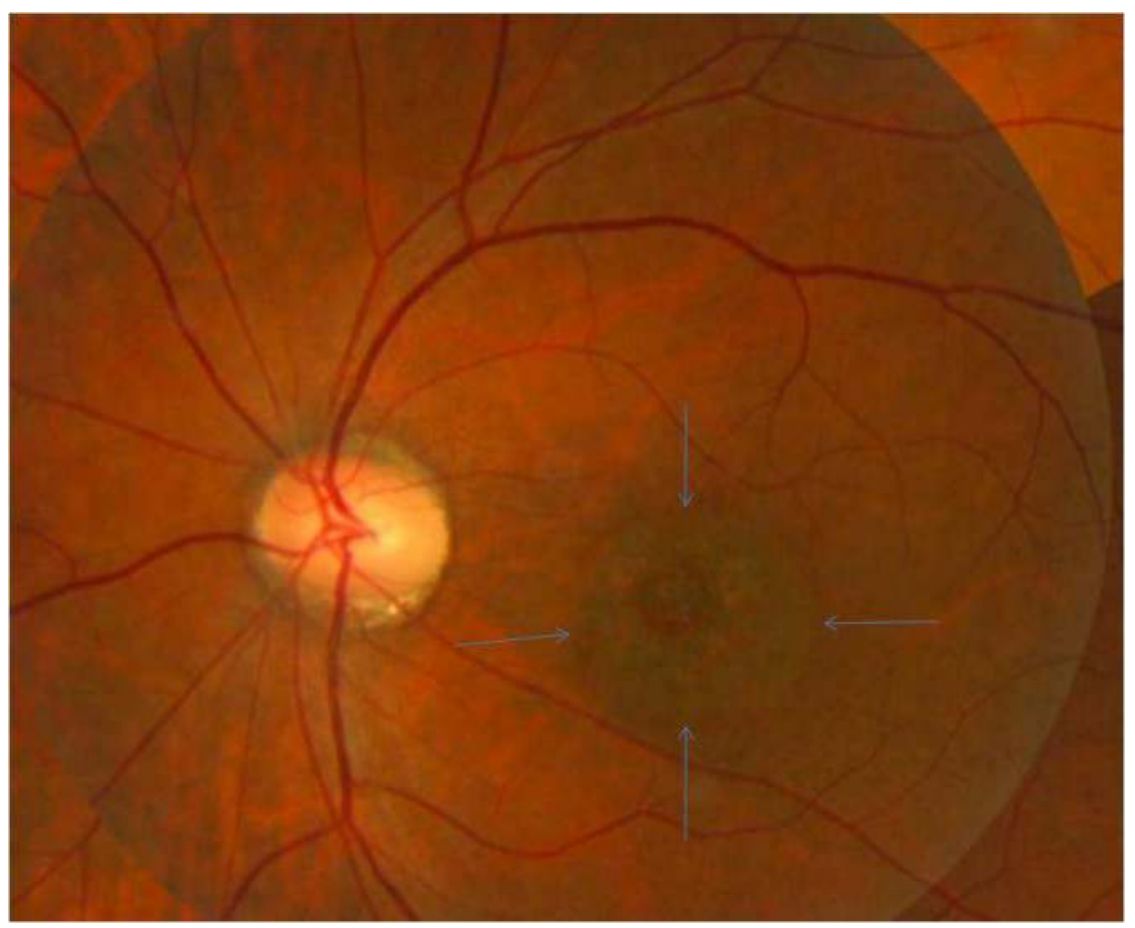

Fig. 7. Fundus photograph of a 17-year-old boy with progressive cone dystrophy. Visual acuity is $20 / 200$. The patient is very photophobic. The macula is modified with a typical bull's eye pattern. The vessels are moderately attenuated. The peripheral retina is normal. An electroretinogram (ERG) is required to confirm that rod responses are within normal values.

The OCT pattern is reversed in cone dystrophies: the disorganization of the IS/OS junction is observed in and limited to the fovea and the perifoveal zone. The thinning of the ONL is progressive and easily recognized in the fovea where the INL and GCL are not present (fig $8,9)$. OCT anomalies are again noted at early stages, and precede the reorganization of the macula on biomicroscopic examination (fig 8). 


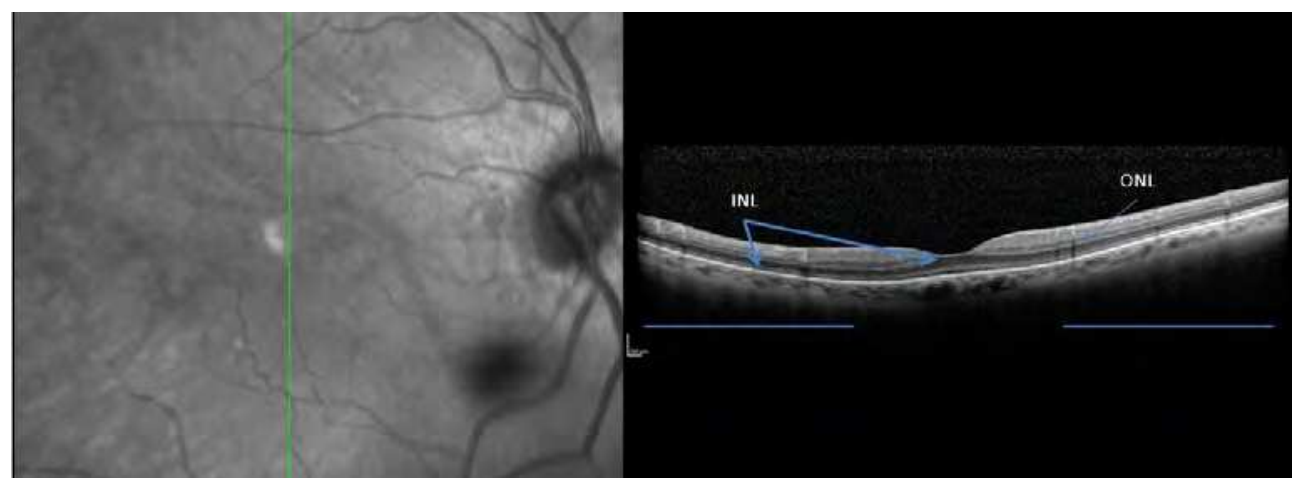

Fig. 8. Red free photography (left) and corresponding OCT scan of a young boy with a visual acuity of 20/200 in both eyes (right). The IS/OS junction is normal in the extrafoveal regions (blue bars). In the central zone, the IS/OS junction is disorganized, and the INL is not as developed as it should be in the foveola (compared with figure 2). The macula appears normal in the red free photograph (left).

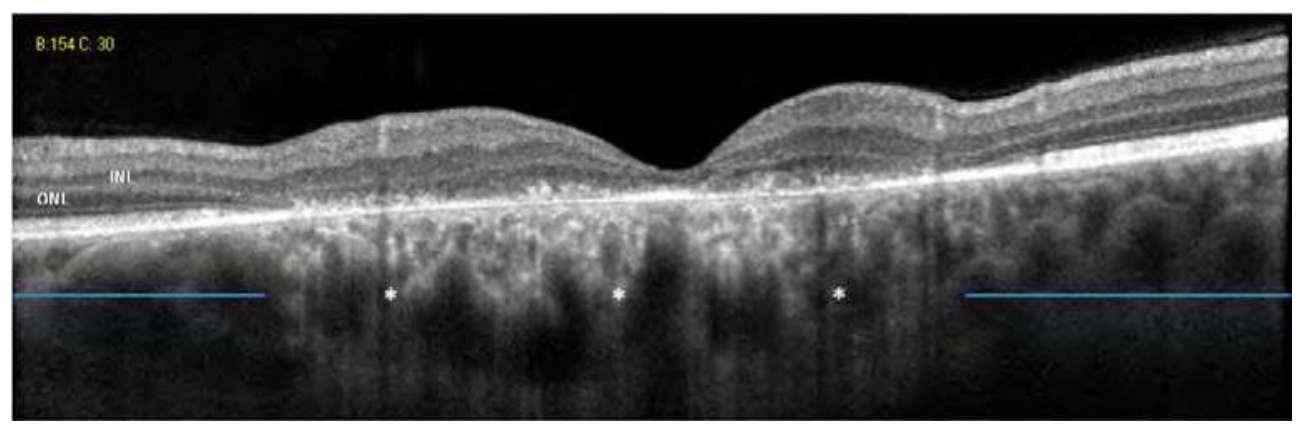

Fig. 9. OCT scan at a more advanced stage of cone dystrophy. Outside the fovea (blue bars), the ONL is very thin in comparison with the INL. In the fovea and perifoveal zone (between the blue bars), no IS/OS junction or ELM is observed. Note the hyperreflective signal of the choroid, related to RPE loss $\left(^{*}\right)$.

\subsection{Cone-rod dystrophy}

Cone-rod dystrophies (CRDs) belong to the group of pigmentary retinopathies (fig 10). They are characterized by the progressive damage of photoreceptors, predominantly cones. CRDs are remarkable for the precocity of the loss of visual acuity and the rapid evolution towards central and peripheral visual loss. The evolutive sequence here is the inverse of that in retinitis pigmentosa: night blindness and anomalies of the peripheral visual field appear after macular damage, and may remain of in the background. An ERG is unavoidable to distinguish the first stages of a CRD, in which the patient may not mention night blindness or problems with the peripheral visual field, from pure maculopathies (including cone dystrophies), which pose no threat to peripheral vision. The frequency of CRDs $(1 / 40000)$ is ten times less than that of retinitis pigmentosa (RCD). 

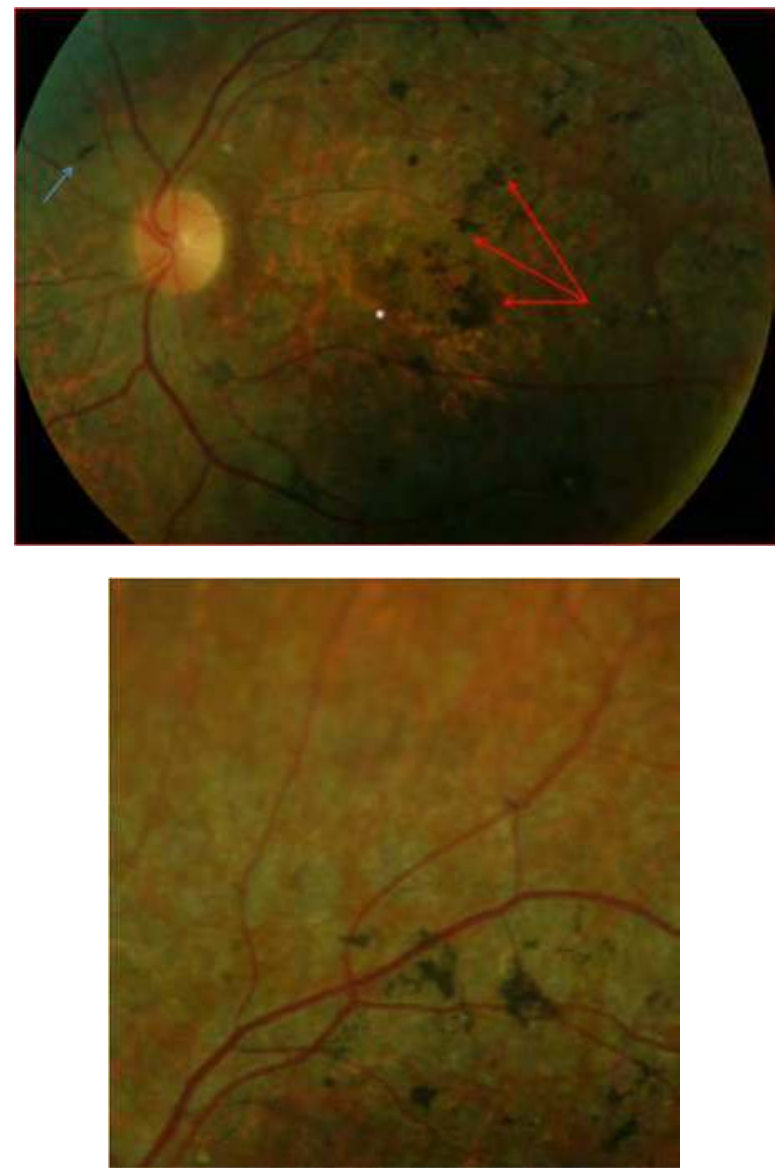

Fig. 10. Fundus of a 17-year-old girl with CRD linked to $A B C A 4$ (the Stargardt gene). Note the presence of pigment deposits of various shapes (red arrows) in the posterior pole with atrophy of the retina (left). The retina appears less damaged in the periphery (right).

The thinning of the neurosensory retina and the discontinuity or loss of the IS/OS junction are very early signs, occurring before any macular reorganization (fig 11). The loss of photoreceptors may predominate in the foveolar or perifoveolar zones (CRD related to $A B C A 4)$ or be diffuse as in retinitis pigmentosa. This is different from the case in pure cone dystrophies, in which the photoreceptor layer is still present and continues beyond the perifoveolar zone.

\subsection{Final stages of RP, COD and CRD}

The IS/OS junction and the EML are not observed and the ONL is extremely thin or even absent. Indeed, RP and CRD display the same pattern at this stage (fig 12). Cone dystrophies can be separated from CRD or RP, based on the fact that the IS/OS junction is still preserved outside the macula (fig 13). 


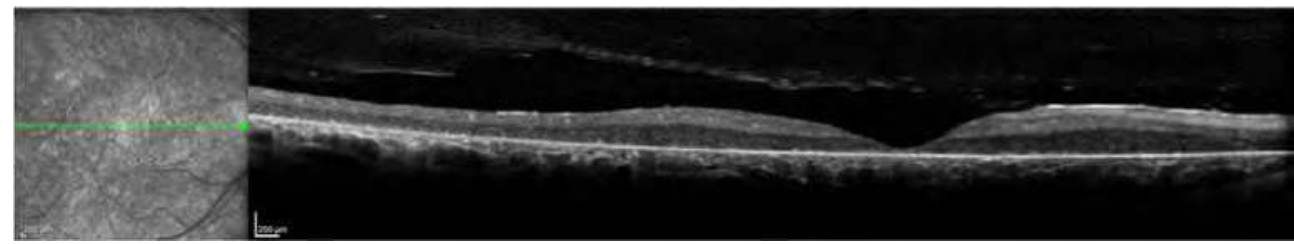

Fig. 12. Red free photography (left) and corresponding OCT, final stage of retinitis pigmentosa or cone-rod dystrophy. No IS/OS junction, EML or ONL can be observed at any point of the section.

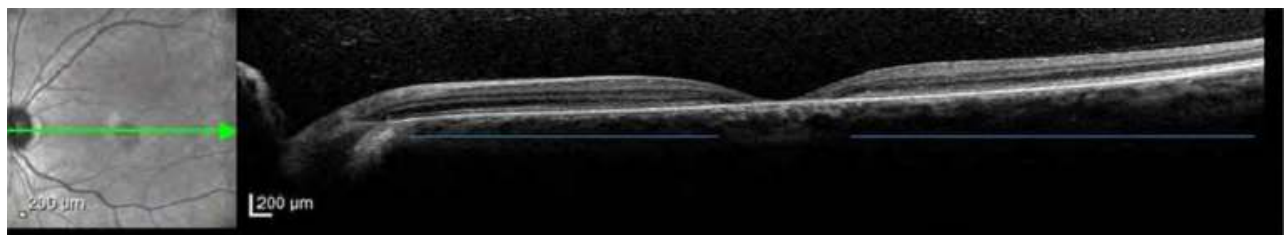

Fig. 13. Red free photography (left) and corresponding OCT, final stage of a cone dystrophy. Visual acuity is 20/100 in both eyes. The macula is atrophic. The defect is small, limited to the fovea (between blue bars). The retina is thin outside the fovea despite the presence of the IS/OS junction. Note that the INL and ONL are thin.

\subsection{Macular edema in RP}

The principal complication is the occurrence of macular edema, with an estimated frequency of $50 \%$ for autosomal dominant forms and $30 \%$ for recessive forms. Macular edema has been rarely observed in X-linked forms. OCT is relevant to the diagnosis of macular edema and for the evaluation of the therapeutic effectiveness of acetazolamide in patients with RP. The prognosis depends on the preservation of the IS/OS junction (fig 14-16) below the central cystic space corresponding to the foveola (Hyewon et al, 2006; Sandberg et al, 2008).

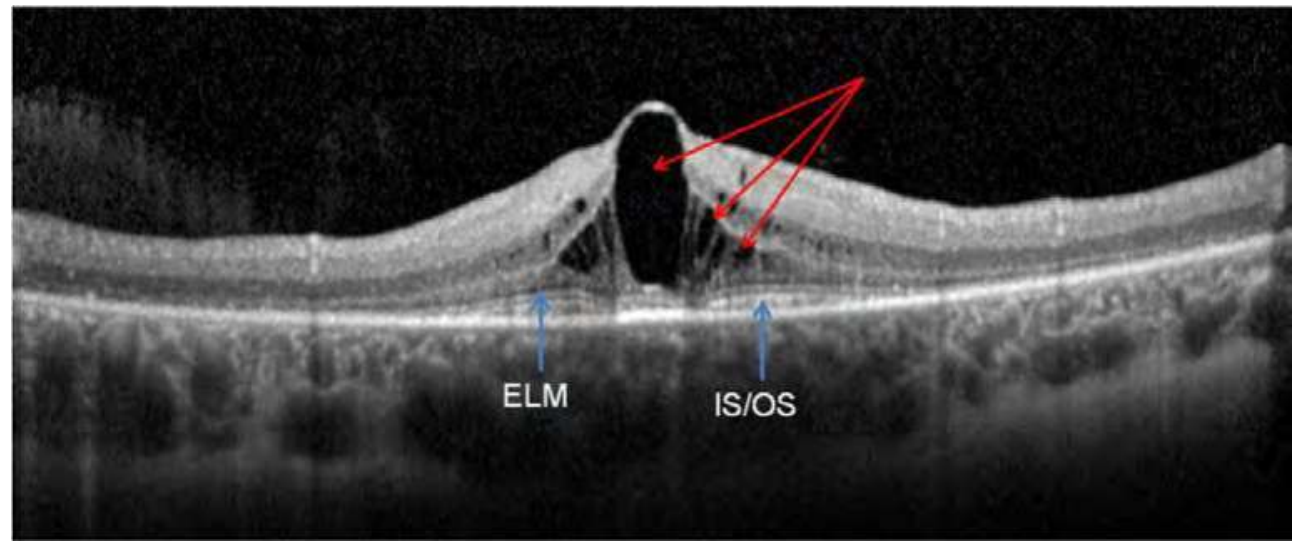

Fig. 14. Cystoid macular edema with large intraretinal cystic spaces. The IS/OS junction is present below the edema, with the exception of the central cyst. 


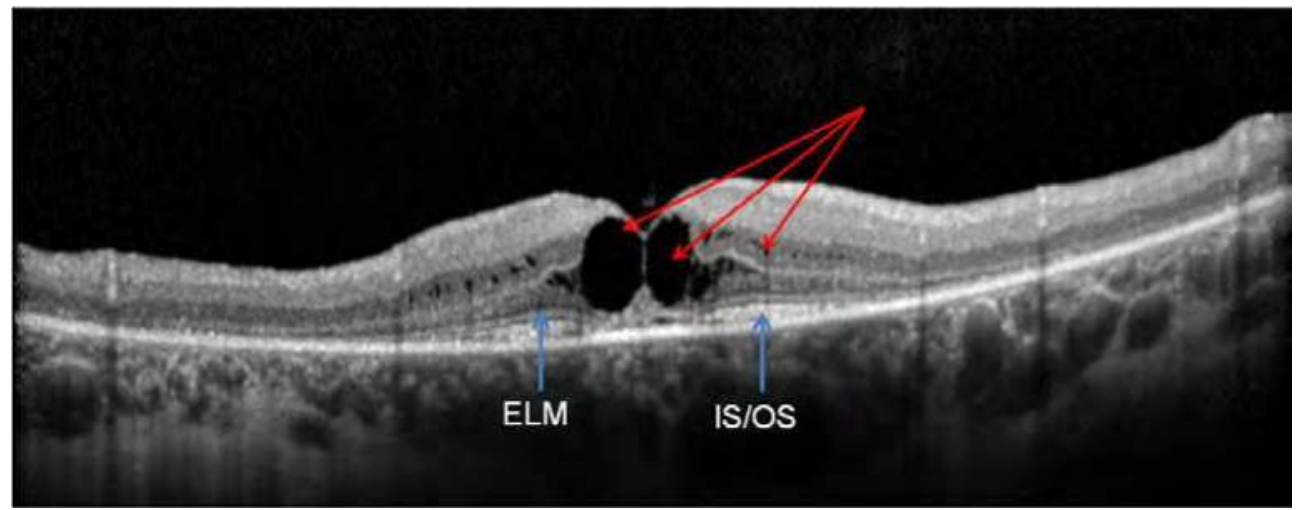

Fig. 15. Same patient as in figure 14, other eye. The thickening of the fovea is less pronounced than in the right eye. The IS/OS junction is present below the edema, with the exception of the two central cysts.

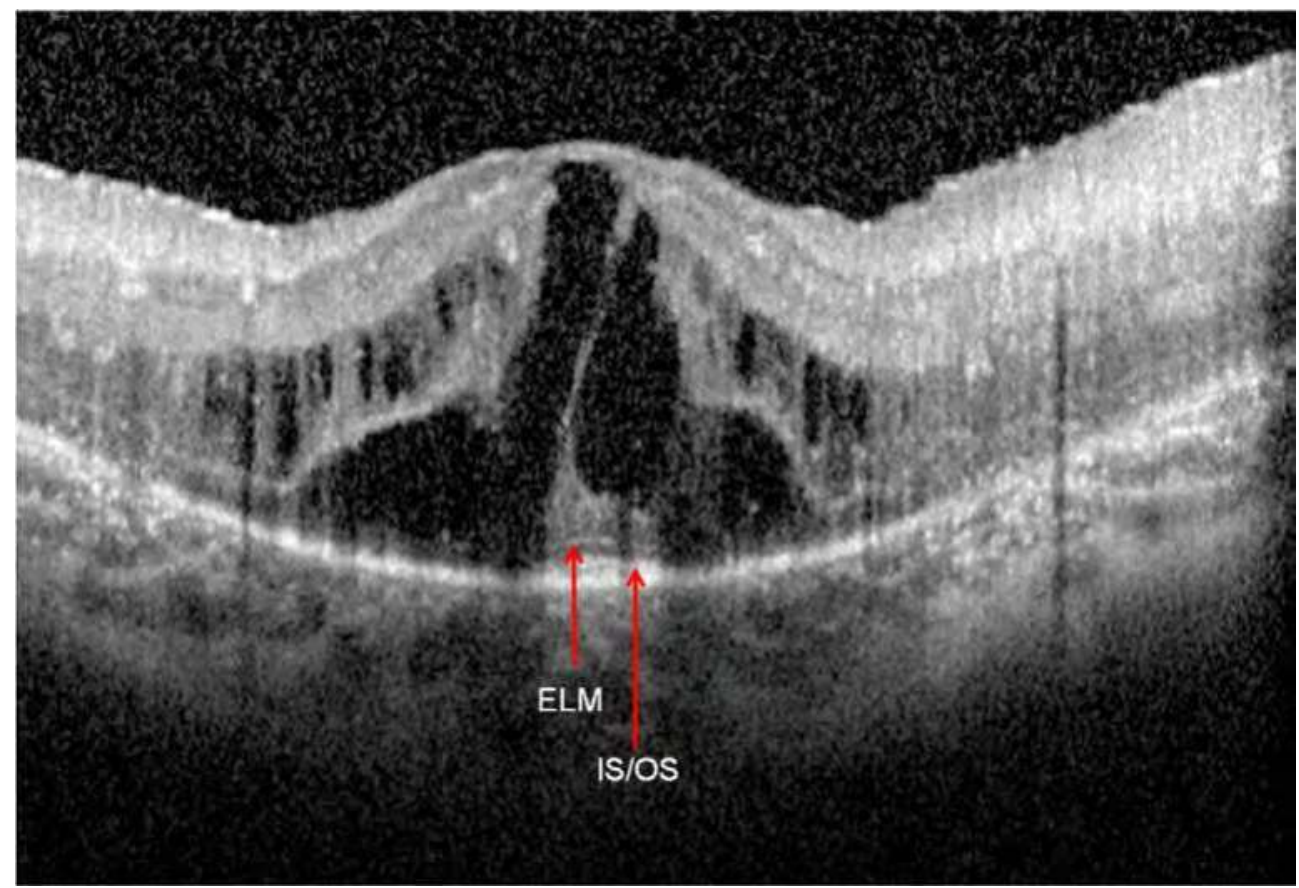

Fig. 16. Severe cystoid macular edema with preserved IS/OS junction below the central cystic space. Visual acuity could be improved by treatment with acetazolamide.

\section{Inherited macular diseases}

This term is used to indicate maculopathies with or without autofluorescent deposits and those with drusen (or drusen-like lesions). These maculopathies are for the most part non- 
syndromic. Photophobia, early anomalies of color vision and a decrease in central vision are the principal criteria characterizing these maculopathies. These signs are present to varying degrees: photophobia is a major sign in cone dystrophy, but less obvious in Best disease. Neither night blindness nor any substantial reduction in the peripheral visual field is seen. The loss of visual acuity may be asymmetric but the lesions are symmetric, except in vitelliform dystrophies and pattern dystrophies.

\subsection{Stargardt disease}

Described by Stargardt in 1909, Stargardt disease is a bilateral symmetric maculopathy, characterized by the presence of a variable number of fish-scale shaped, yellowish, autofluorescent deposits (fig 17,18). This maculopathy, which has an autosomal recessive transmission linked to the $A B C A 4$ gene, is the most frequent of juvenile maculopathies with a prevalence of 1 in 10000 , and represents 7\% of hereditary diseases. In Stargardt disease, the degeneration of photoreceptors (PR) expressing a genetic abnormality is thus secondary to the loss of pigment epithelial cells. The preferential macular damage is explained by the high density of cones and rods in the perifoveolar and foveolar zones. There is an early and severe loss of visual acuity, even if the reorganization of the fundus appears to be slight. Visual acuity stabilizes at around 20/200 to 20/400.

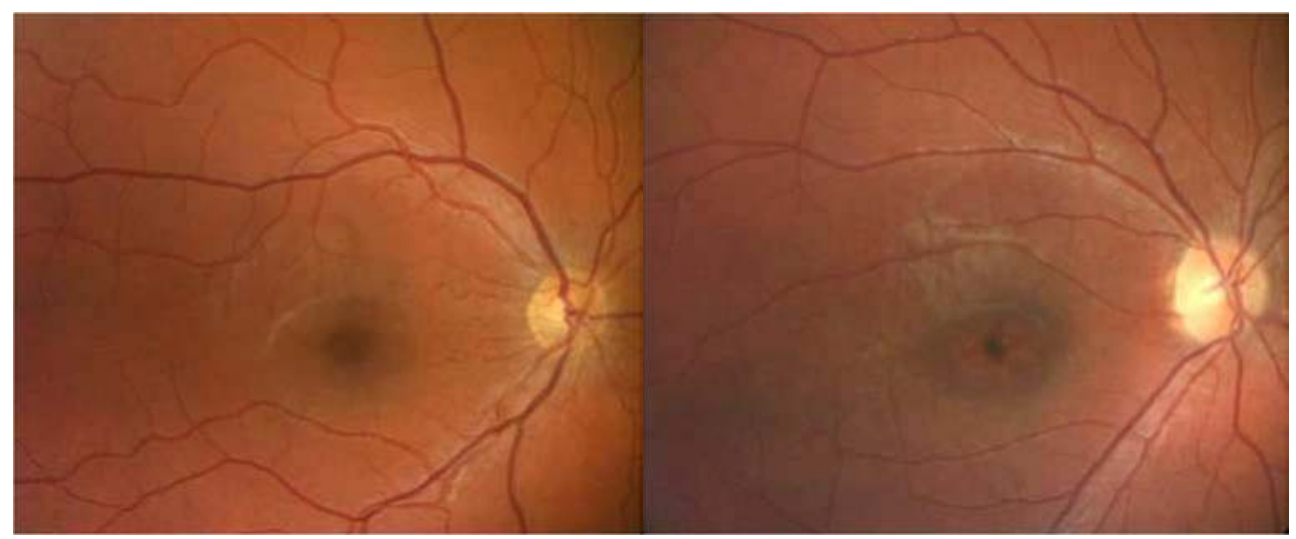

Fig. 17. Fundus photographs at different stages of Stargardt disease. Left: the macula appears normal in spite of a visual acuity of 20/100 in both eyes in a 12-year-old child. Note the absence of retinal flecks. Right: Oval reorganization of the macula (dark in appearance) along the major horizontal axis in a 9-year-old child with visual acuity limited to 20/200 in both eyes. Visual acuity was at 20/30 at the end of preschool. A few rounded yellowish deposits can be seen temporally.

OCT anomalies occur very early in Stargardt disease including loss of the foveal clivus, discontinuity or loss of the photoreceptor layer (Ergun et al, 2005; Querques et al, 2006). They precede the occurrence of fundus abnormalities. As the zone with the highest density of photoreceptors, the perifoveolar zone is preferentially affected. Thus, OCT reveals a premature loss of the clivus and the photoreceptor layer throughout the zone of macular reorganization defined by autofluorescence images, with a thinning of the retina (fig 19-22). This is very different from the aspect of cone dystrophies in OCT (initial and predominant 


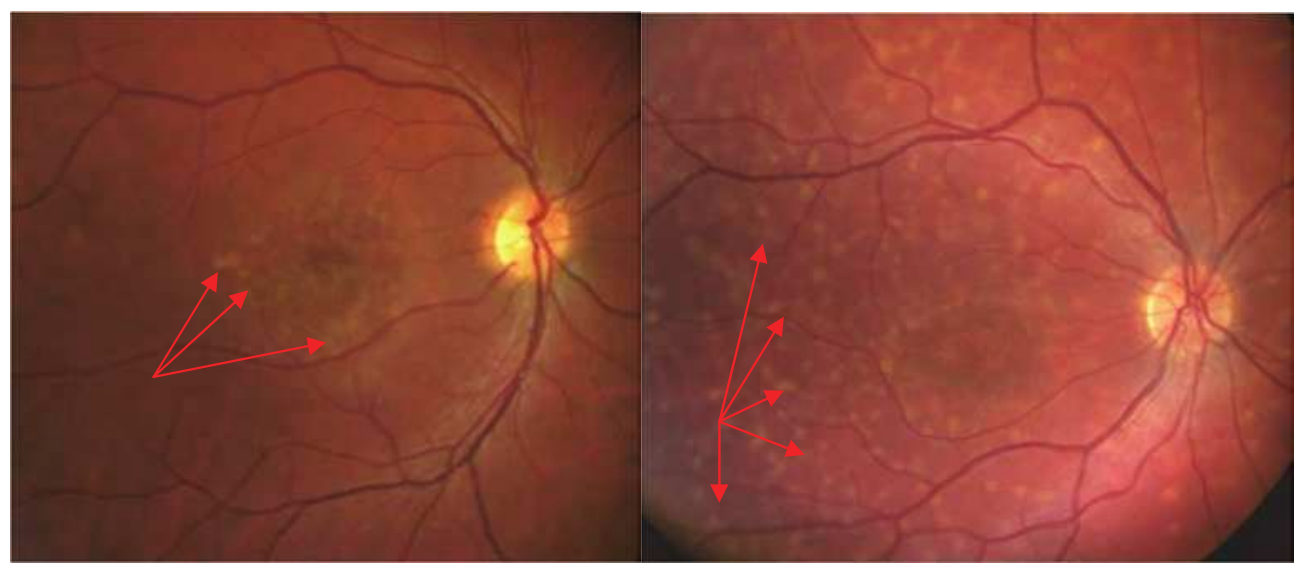

Fig. 18. Left: Macular reorganizations associated with yellowish perifoveolar retinal flecks immediately reminiscent of Stargardt disease in a 19-year-old subject with a visual acuity of 20/40 P2. Right: A 24-year-old subject with a visual acuity of 20/200 in both eyes. There are abundant yellow retinal specks spreading throughout the posterior pole. The macula is extremely disorganized with zones of perifoveolar atrophy.

damage of the retrofoveolar PR layer). Visual acuity is correlated more closely with PR loss than with retinal thinning as measured by OCT. Retinal flecks have the appearance of hyperreflective deposits apposed to the pigment epithelium, or more rarely, further away in the outer nuclear layer [5]. Type 1 flecks are dome-shaped deposits located within the retinal pigment epithelium and the outer segment of photoreceptors (fig 23). Type 2 flecks are deposits involving the outer nuclear layer as well. They are encountered in advanced or severe stages, with the loss of the photoreceptor layer within the central foveal zone.

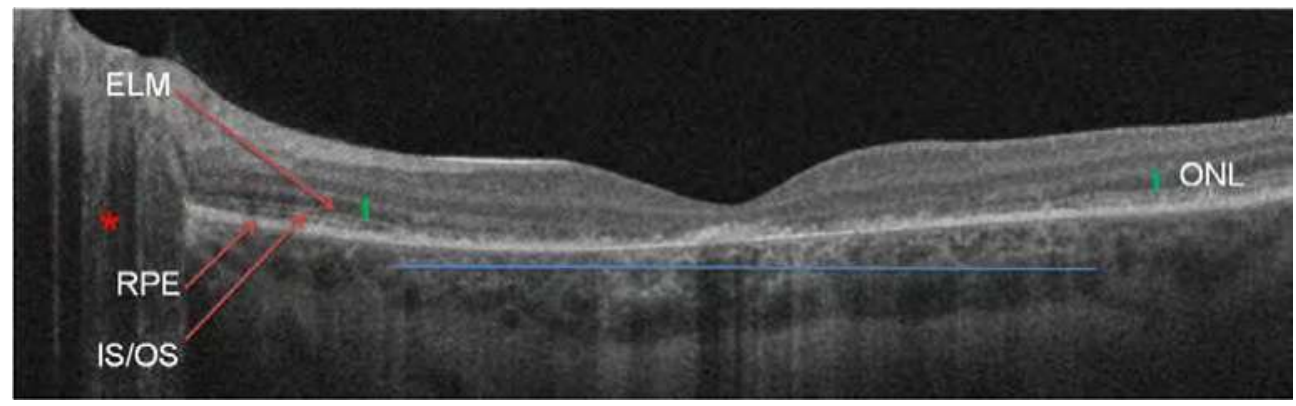

Fig. 19. Horizontal OCT section through the fovea and the optic nerve head. The following elements should be noted: alterations in the RPE and the OS/IS junction, loss of relief of the clivus, thinning of the retina. These alterations reflect the loss of pigment epithelial cells, followed by photoreceptors. In the macular zone (horizontal blue line) the loss of photoreceptors is severe and reflected by the disappearance of the IS/OS junction line, as well as the absence of the outer nuclear layer (ONL, i.e. the layer of photoreceptor nuclei). Retinal thickness is reduced in accordance with the disappearance of the outer layers of the retina. These anomalies are obvious and severe, although the fundus appears normal. 


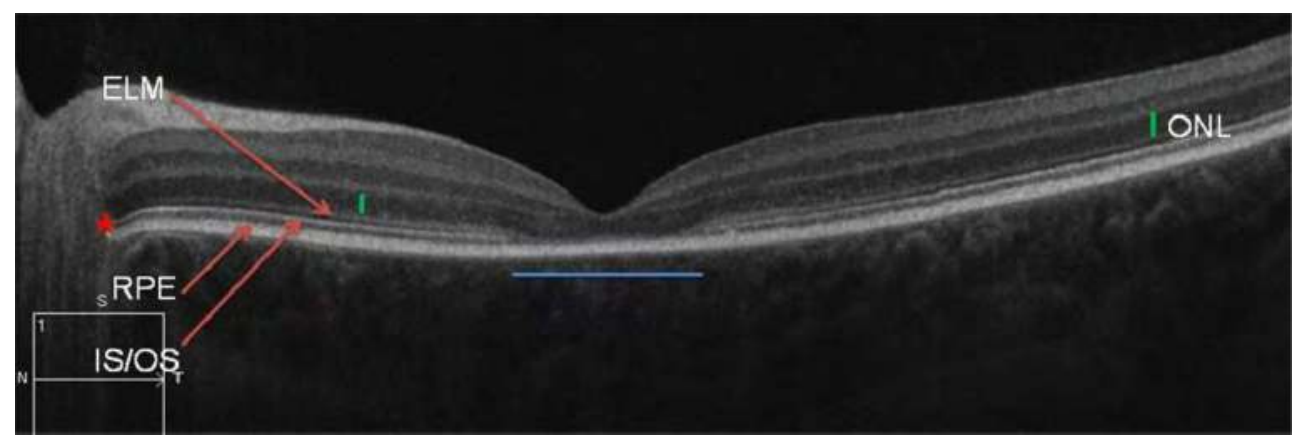

Fig. 20. Disappearance of the IS/OS junction line of photoreceptors, and the absence of the outer nuclear layer (ONL, i.e. the layer of photoreceptor nuclei) in the limited subfoveal zone.
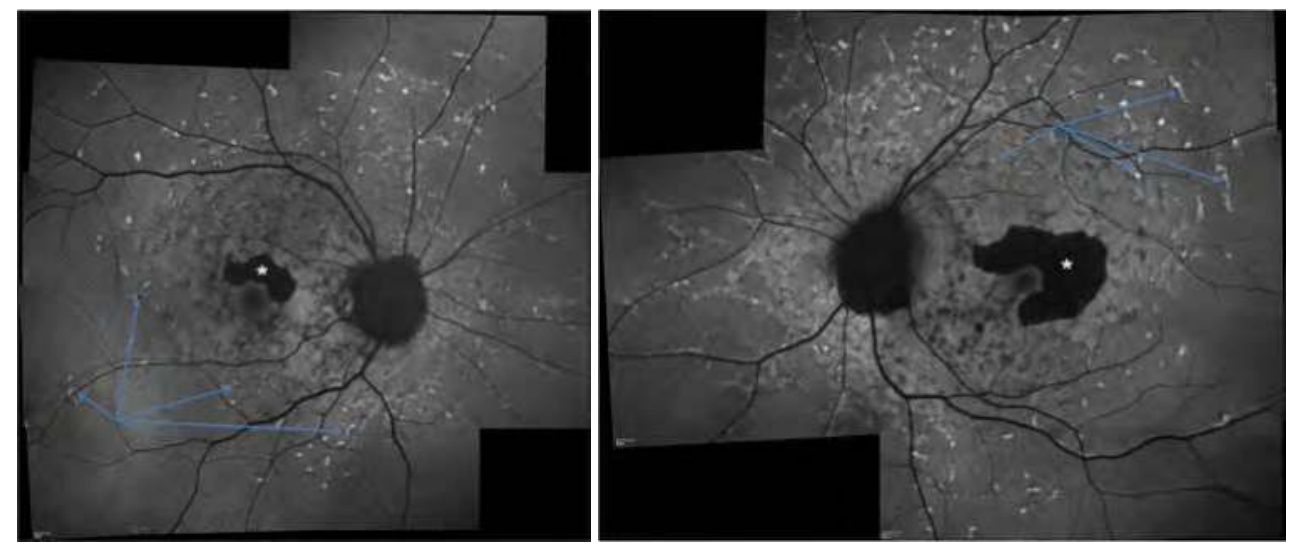

Fig. 21. Late-onset Stargardt disease with perifoveolar damage. Autofluorescence images: The bilateral and symmetric retinal flecks are autofluorescent (arrows). The perifoveolar atrophy appears dark black $\left(^{*}\right)$, and spares the fovea in both eyes.

\subsection{Best disease}

Initially described by Adams in 1883 and revisited by Best in 1905 from a genetic point of view (autosomal dominant transmission), Best disease is characterized by the present of autofluorescent vitelline deposits ("vitelliform deposits"). The sequence of evolution of the deposits is stereotypic (fig 24), from their appearance to the fragmentation of the material until its reabsorption. The deposits, single or multiple, macular or extramacular, can differ in their evolutive stage from one eye to the other, or even within the same eye. This asymmetry, both in the size of the lesions and in their stage, is particularly evocative of the vitelliform dystrophies, which are the only asymmetric unilateral or bilateral hereditary maculopathies. Best disease, the second most-widespread juvenile maculopathy, is linked to the BEST1 gene. The visual prognosis for this dystrophy is relatively satisfactory, unlike that of other hereditary disorders: a majority of patients conserve visual acuity in one of the two eyes $(71 \%$ of cases). 

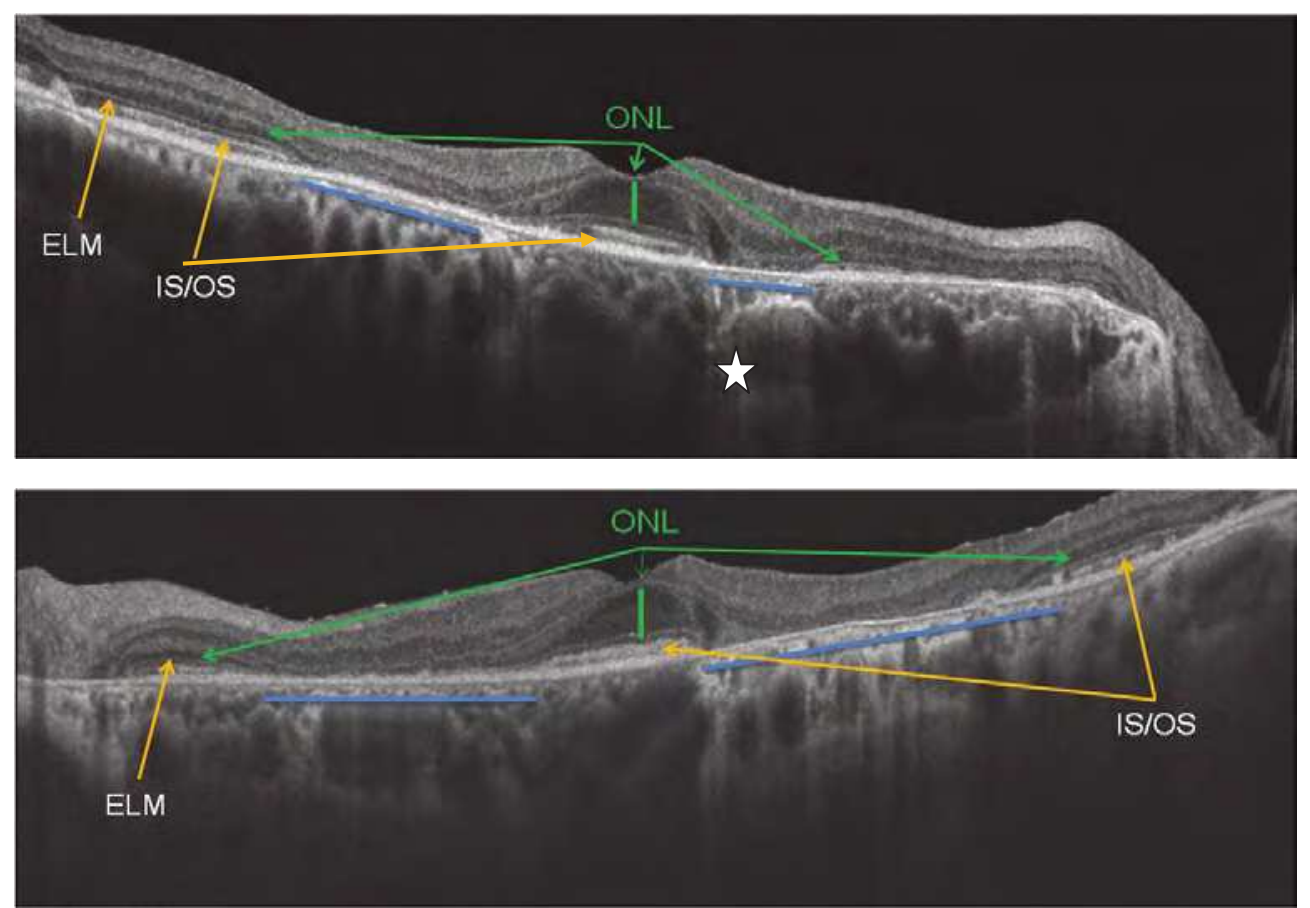

Fig. 22. Late-onset Stargardt disease with perifoveolar damage (same patient as figure 21). Top: right eye. Bottom: left eye. The IS/OS layer is totally absent in the perifoveolar zone only (blue line). The choroid displays a hyperreflective signal (star). The thickness of the fovea is normal, with a well-preserved photoreceptor layer, accounting for the conservation of visual acuity.

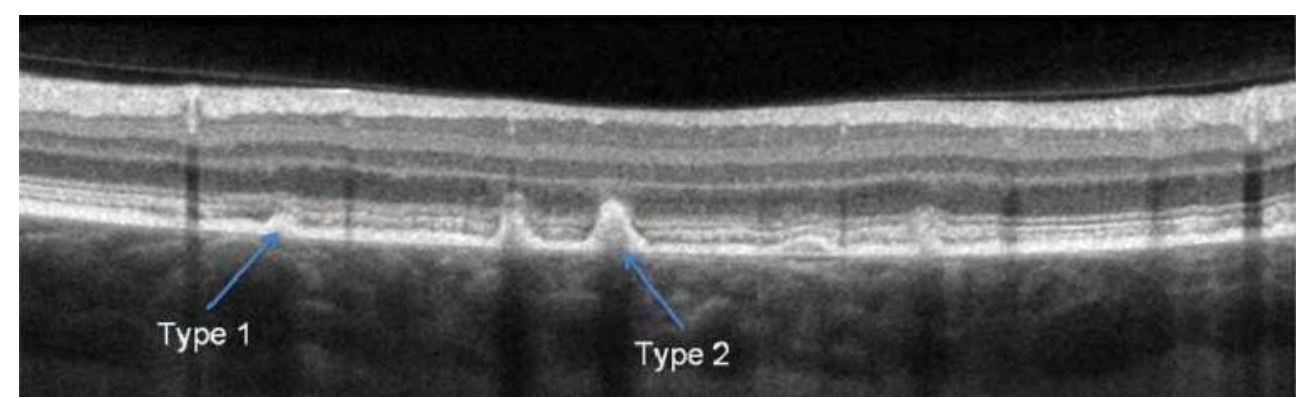

Fig. 23. Retinal flecks in Stargardt disease. Type 1 flecks are dome-shaped deposits located in the retinal pigment epithelial layer and the outer segment of the photoreceptors. Type 2 deposits also involve the outer nuclear layer.

In OCT, the vitelline material appears as a convex/dome-shaped, hyperreflective and homogenous lesion (Fig 25), located below the hyperreflective photoreceptor layer (Ferrara et al, 2010). 


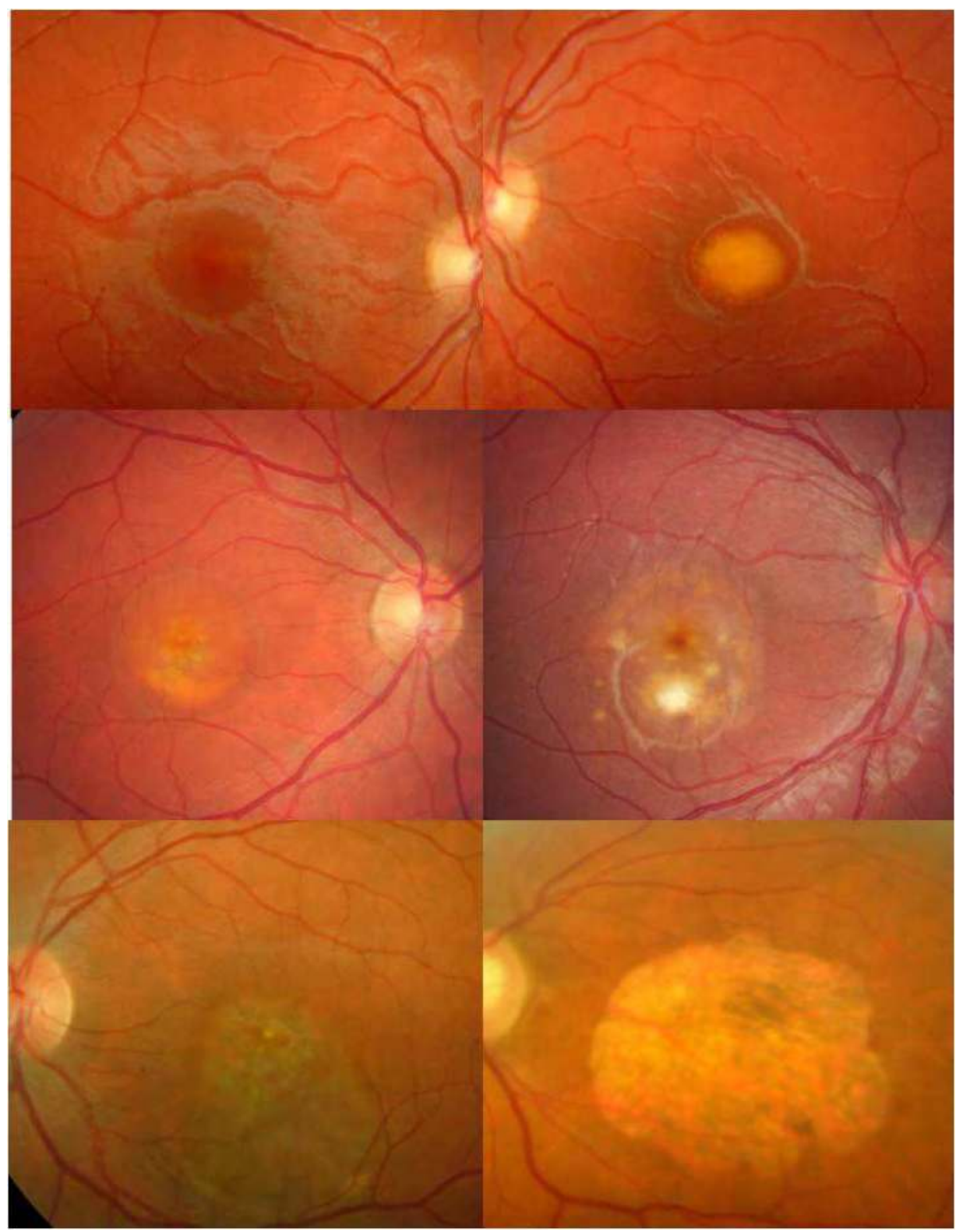

Fig. 24. Evolutive sequence of vitelliform lesions. A. Previtelliform stage. B. Vitelliform stage. C and D. Scrambled egg stage with (C) or without hypopyon (D). E. RPE alterations; the vitelline lesion has been resorbed. F. Atrophic stage. 


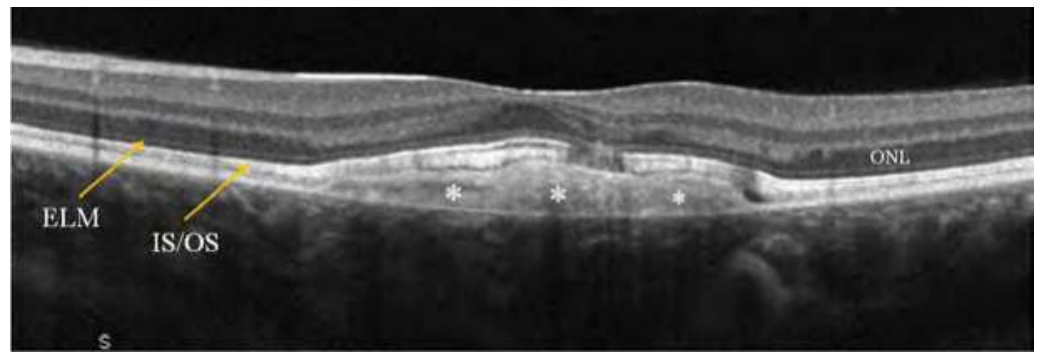

Fig. 25. Best disease, vitelliform stage. The subfoveal vitelliform deposit $\left(^{*}\right)$ is homogenous, situated at the level of the pigment epithelium and above it, touching the inner/outer segment (IS/OS) junction line and the external limiting membrane (ELM).

At the fragmentation stage, differences in the reflectivity of the material and the appearance of dark, optically empty zones resembling a false serous retinal detachment can be noted (fig 26-27). This last, pseudo-retinal detachment-like appearance associated with vitelline material should not be taken for a neovascular complication. Only exudative intraretinal modifications are a sign of neovascularization. The photoreceptor layer may be discontinuous with diminished visual acuity, or conserved with normal visual acuity.

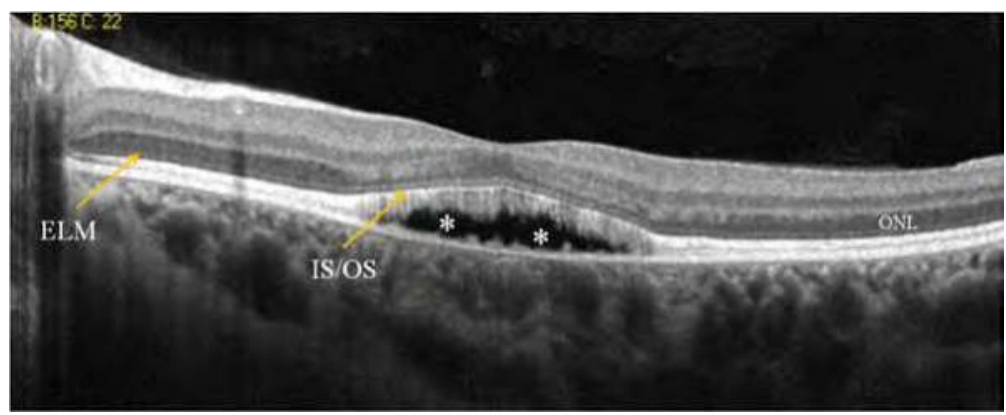

Fig. 26. Best disease, scrambled-egg stage. The deposit is optically empty and dark. Note the perfect conservation of the photoreceptor layer, including above the vitelline material. Visual acuity is $20 / 20$.

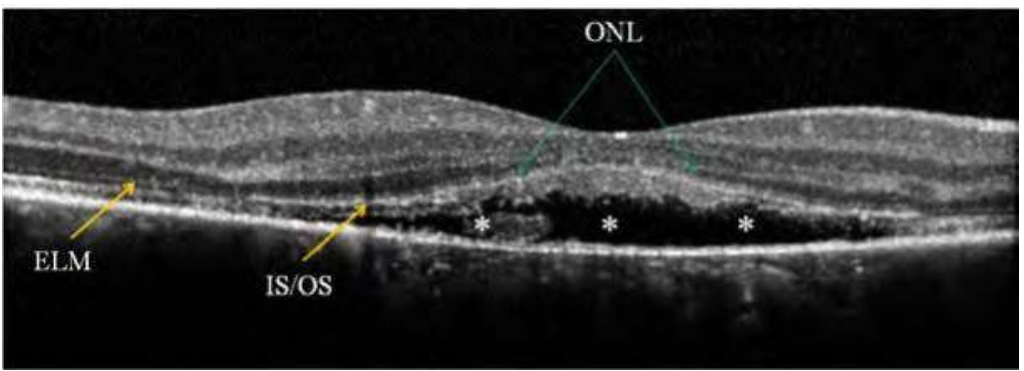

Fig. 27. Best disease, scrambled-egg stage. Visual acuity is 30/60. Extensive scrambling of the lesion except at the left extremity. The inner/outer segment (IS/OS) junction is altered and the outer nuclear layer has disappeared. 
At later stages of atrophy and/or fibrosis, the lesion is hyperreflective, the overlying retina is disorganized and thin, and the photoreceptor layer may be completely absent (fig 28).

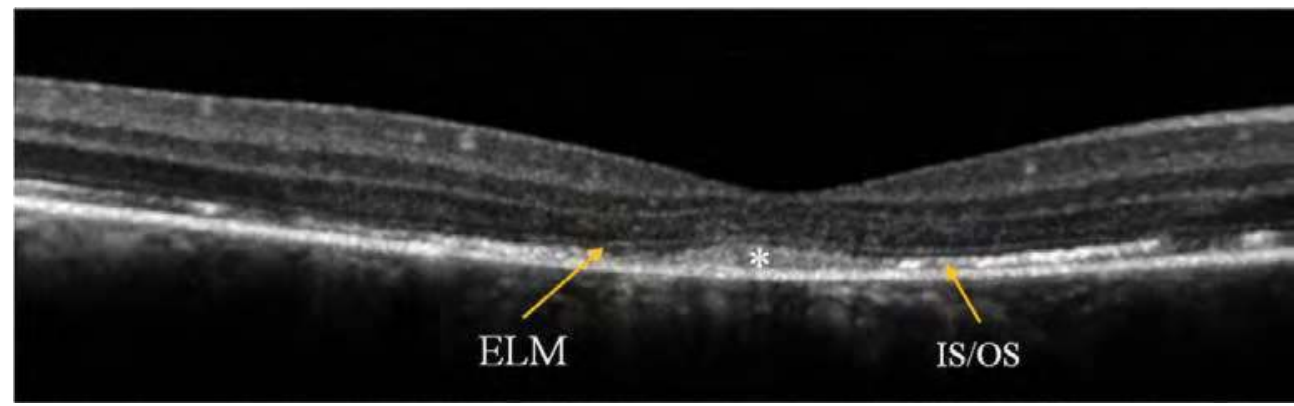

Fig. 28. Best disease, RPE alterations. Visual acuity is 20/30. The external limiting membrane is preserved (ELM). The subfoveal IS/OS line is ill-defined. The grey structure $\left(^{*}\right)$ corresponds to residual vitelline material.

\subsection{Juvenile $X$-linked retinoschisis}

X-linked juvenile retinoschisis is the most frequent form of macular retinoschisis (fig 29). The diagnostic signs are simple, with an appearance characteristic of macular schisis in OCT, confirmed by an electroretinogram (ERG). Prevalence is variable, with geographical differences ranging from 1/5000 in Finland to 1/25 000 elsewhere. X-linked juvenile retinoschisis is associated with mutations of the RS1 gene (6 exons). This gene, expressed by photoreceptors and bipolar cells, codes for retinoschisin, a protein involved in adhesion and cohesion of the retina. This function depends on a conserved region of the protein, the discoidin domain, encoded by exons 4 to 6 . Macular retinoschisis results in the progressive thinning of the retina with a slow and variable decrease in visual acuity after 50 years of age. Macular atrophy is the last stage.

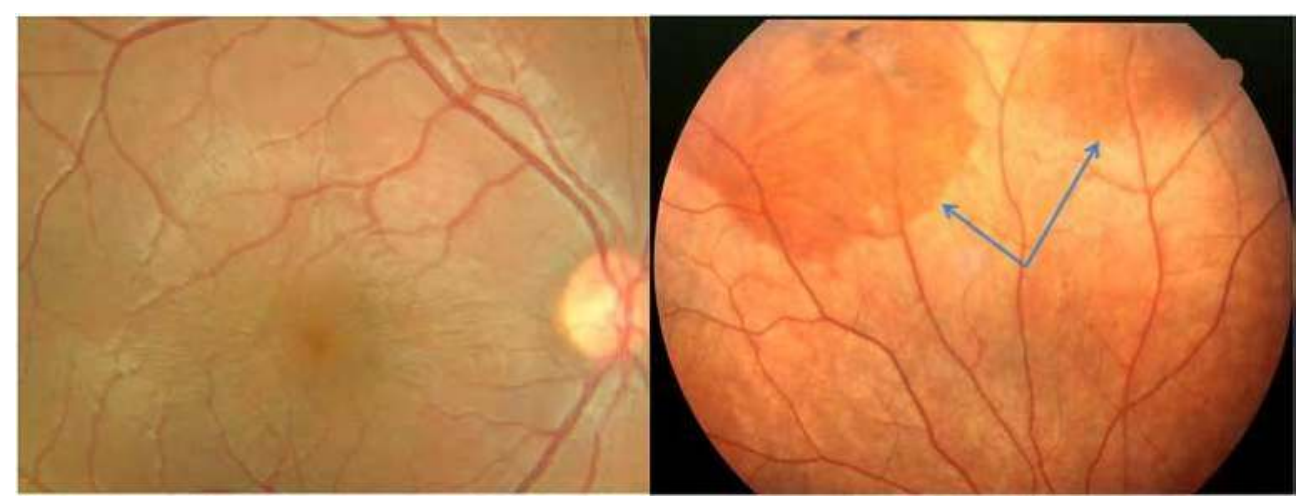

Fig. 29. X-linked juvenile retinoschisis. Left : Characteristic bilateral and symmetric wrinkling of the retina resembling the spokes of a wheel in a 7-year-old boy. Right: the two rounded darker-colored zones in the superior periphery are flat peripheral retinoschisis. 
Spectral OCT is unavoidable for the visualization of a pathognomonic pattern of macular cysts that can extend up to the mid-periphery (Yu et al, 2010). Within the retina, the cysts can extend from the ganglion cell layer to the inner or outer nuclear layer (fig 30, 31). In the late stage of the disease, the zone corresponding to the schisis is thin or atrophic, and its appearance is aspecific.

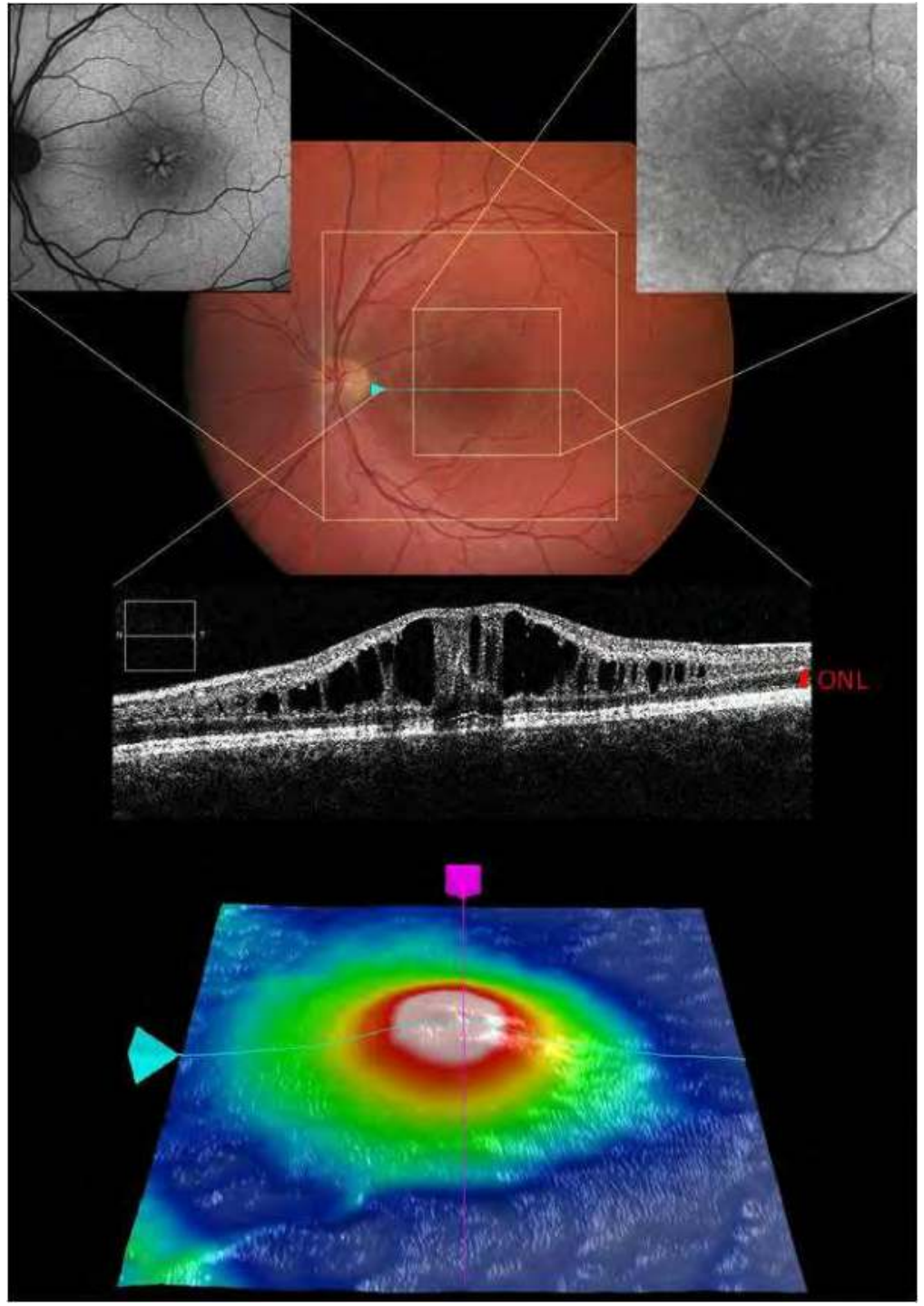

Fig. 30. Spectral-domain OCT of a macular retinoschisis in a young boy. The cysts extend throughout the perimacular retina and the ganglion cell layer up to the outer nuclear layer, which is not involved. Insets showing the typical autofluorescent pattern of retinoschisis (two top greyscale images; the one on the right is a magnification of the macular schisis). 


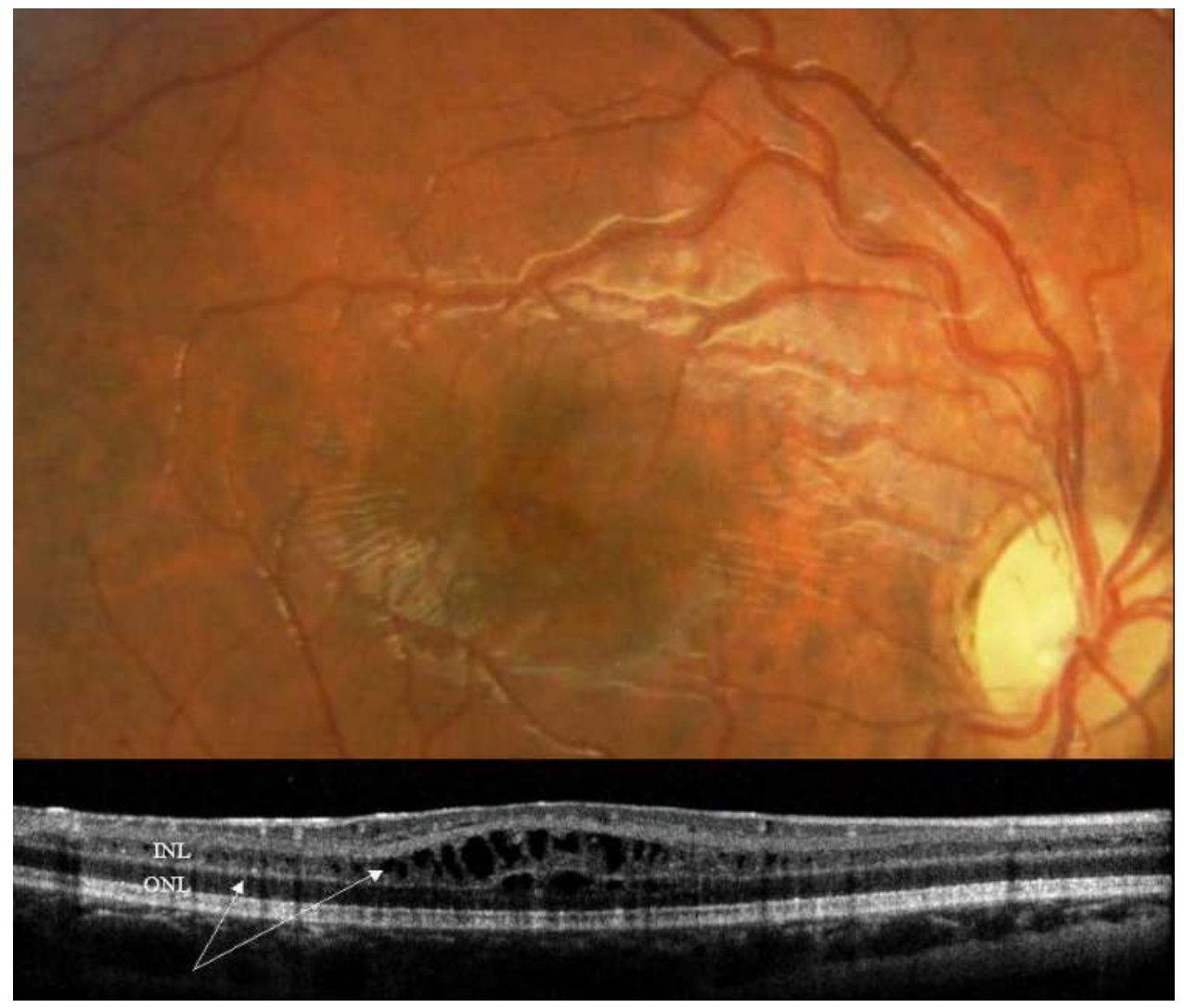

Fig. 31. Spectral OCT pattern of an X-linked retinoschisis in a young boy. The entire retina is affected, and cysts (arrows) have developed within the inner (INL) and outer nuclear layers (ONL).

\subsection{Malattia Leventinese}

Malattia Leventinese was described by Vogt in 1925 (in families in the Leventine valley in Switzerland). However, the first cases were reported by Doyne in 1899 (Doyne's honeycomb retinal dystrophy). In addition to the autosomal dominant transmission of the disorder, drusen in Malattia Leventinese have specific features depending on the age of onset (child or young adult), their localization, their radial arrangement in the periphery, and their confluency (fig 32).

This maculopathy is rare, and linked to a mutation in the EFEMP1 gene (EGF containing fibulin-like extracellular matrix protein 1). Visual acuity decreases after 40 years of age, with blindness at around the $7^{\text {th }}$ decade, related to atrophy or to choroidal neovessels [5]. 


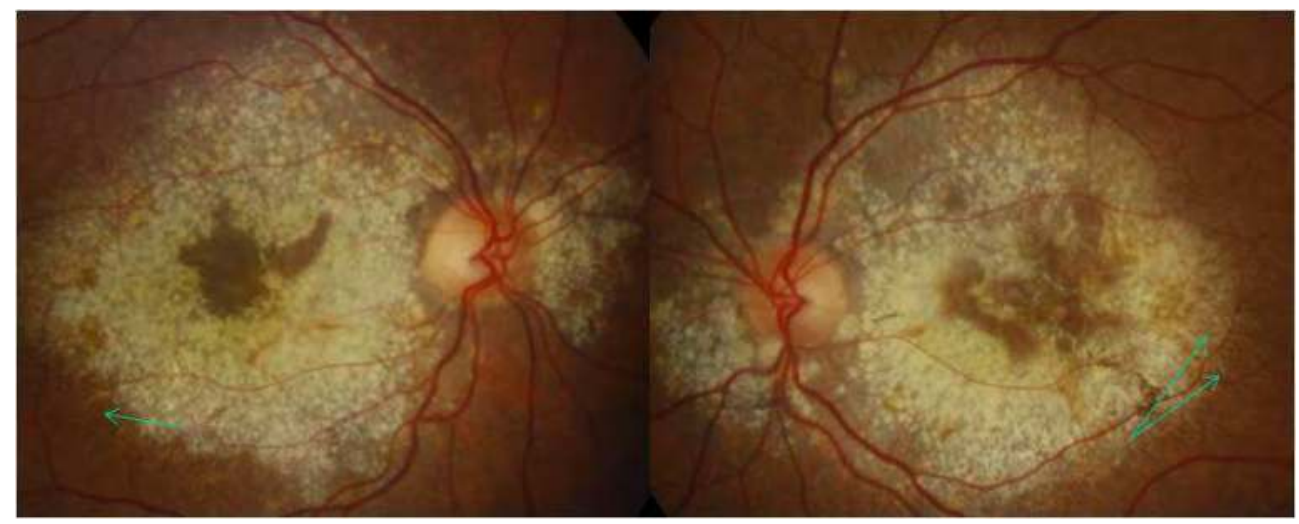

Fig. 32. Typical Malattia Leventinese with a honeycomb-like appearance in a 46-year-old woman with a visual acuity of 20/200 in both eyes. The drusen are dense, with a specific macular and peripapillary topography. Note the radial distribution of the fine peripheral drusen (arrows).

Its appearance in OCT is very specific, with a thick, continuous, homogenous, hyperreflective material between the Bruch membrane and the pigment epithelium (Gerth et al, 2009). These deposits push back and lift the entire retina, which does not show much morphological modification but which becomes wavy (fig 33). The dark cones correspond to alterations of the pigment epithelium. The photoreceptor layer is not altered until later.

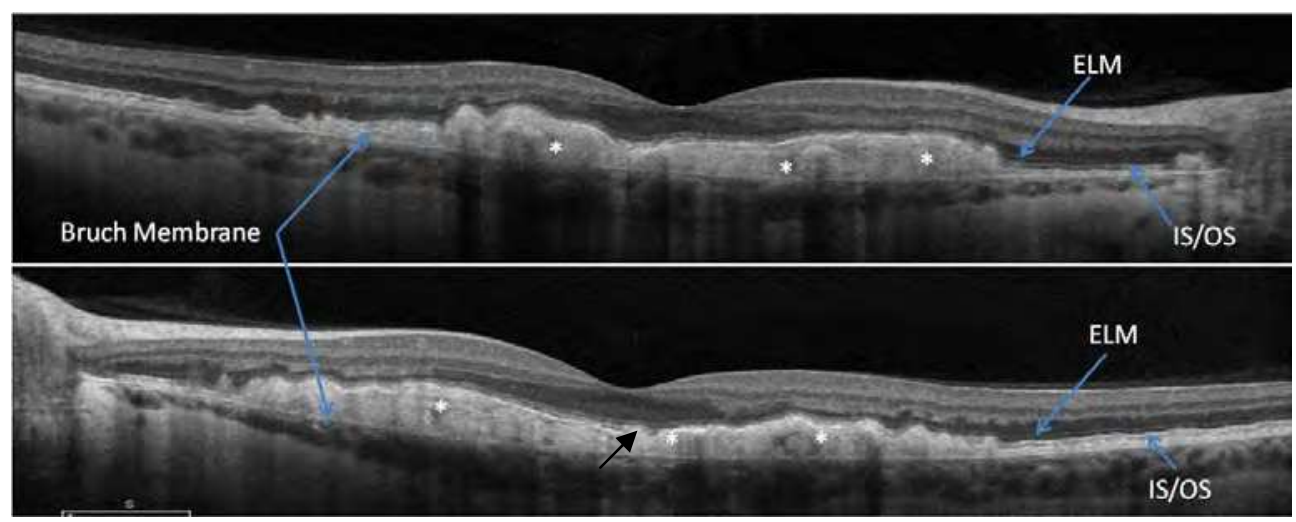

Fig. 33. Malattia Leventinese. OCT scans of a 36-year-old woman. Visual acuity is 20/20 in the right eye, 20/30 in the left eye. The material lies above the Bruch Membrane, and a part of the subfoveal IS/OS line is interrupted in the left eye (bottom, black arrow).

\subsection{Sorsby's fundus dystrophy}

Initially described by Sorsby in 1949, this hereditary autosomal dominant dystrophy is characterized by great clinical heterogeneity. The initial clinical profile can be limited to unilateral choroidal neovascularization, unusual for the age of onset (non-myopic young adults), and associated with a family history of retinal disease. This dystrophy is not limited 
to maculopathy: multiple non-specific lesions appear in varying combinations at the posterior pole and later in the periphery, progressing centrifugally. At a more evolved stage, the damage is bilateral and polymorphic, including diverse lesions at the posterior pole: yellowish-white drusen-like lesions, yellowish plaque-like subretinal deposits, streaks, patches of fibrotic scarring, and in the periphery, abnormal pigmentation and patches of depigmentation or atrophy. This rare dystrophy is linked to a mutation of the TIMP3 gene (tissue inhibitor of metalloprotease 3), expressed by the cells of the pigment epithelium. Choroidal neovessels are the first cause of the reduction in visual acuity in this dystrophy (fig 34). The mean age at which the first eye is affected is 46 years, and 50 years for the second eye. This complication is very frequent ( $60 \%$ of cases) with a bilateral involvement in $50 \%$ of cases. The second factor for the severe loss of visual acuity (less than 20/200) is macular atrophy, which occurs at a mean age of 50 years for the first eye and 52 years for the second.

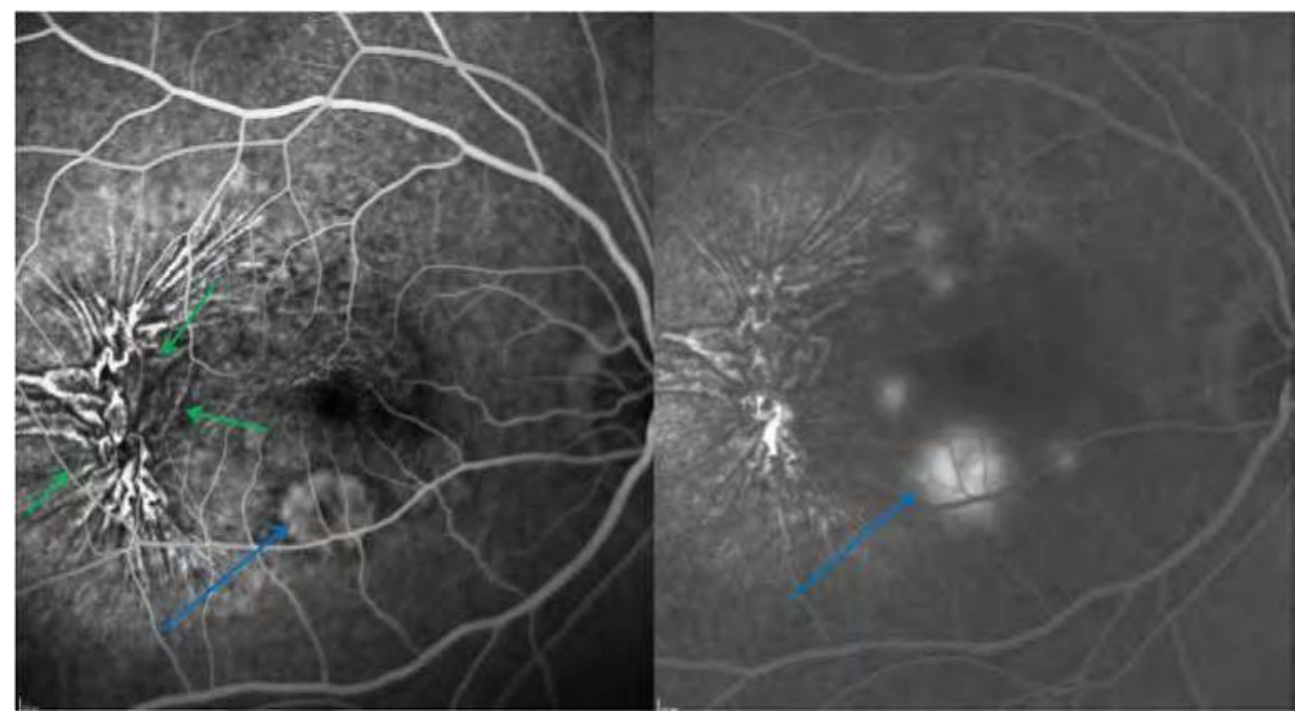

Fig. 34. A 40-year-old man referred for acute visual loss in the right eye due to choroidal neovascularization. The sea-fan-shaped complex is hyperfluorescent in an early frame (left), with diffusion of the fluorescein dye at a later phase (blue arrows). The exudation extends temporally through the retinal pigment epithelium and detaches the overlying retina. There is a contraction of the neovascular net, as revealed by chorioretinal folds (green arrows).

In OCT, the retina has a very particular appearance. Independent of neovascularization (fusiform hyperreflectivity, cavities, serous detachment, detachment of the pigment epithelium), the pigment epithelium-choroid complex is abnormally hyperreflective in certain zones of the posterior pole (Saihan et al, 2009). This hyperreflectivity is characteristic, and different from the abnormal hyperreflectivity of the choroid due to atrophy of the overlying pigment epithelium (fig 35). 


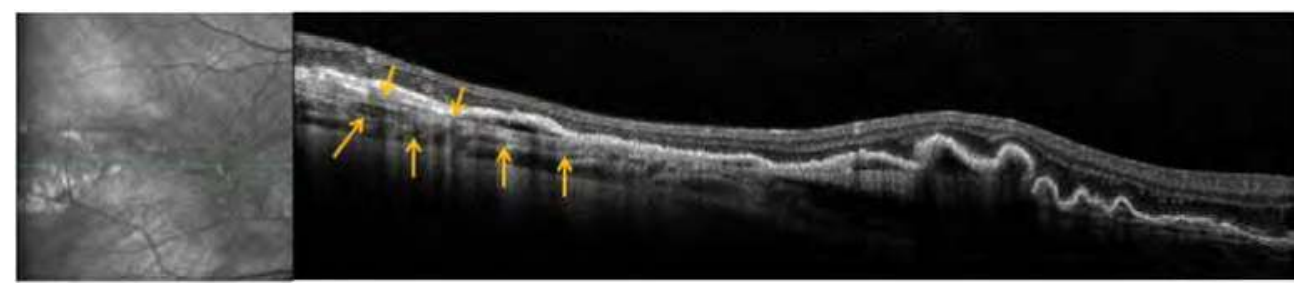

Fig. 35. OCT scan of the patient described in figure 33 demonstrates hyperreflective signals at the level of the retinal pigment epithelium-choroid complex (yellow arrows).

\subsection{North Carolina dystrophy}

North Carolina dystrophy, initially described by Lefler, is a maculopathy that is remarkable for its lack of evolutivity and the relative conservation of visual acuity. Each patient has a characteristic level of visual acuity and type of macular lesion (Grade 1, 2 or 3) that is invariable over time. Only grade 3 lesions are distinctive: the macula is excavated, colobomatous, and includes fibrous tissue and pigmented zones (fig 37). Grade 1 and 2 lesions (drusen) are non-specific, but their age of onset is very unusual [1-3]. This autosomal dominant dystrophy is very rare. A first identified locus, 6q14-q16.4, is thought to be involved in the approximately 5000 American cases (from an ancestral mutation carried by three Irish brothers who emigrated to North Carolina).

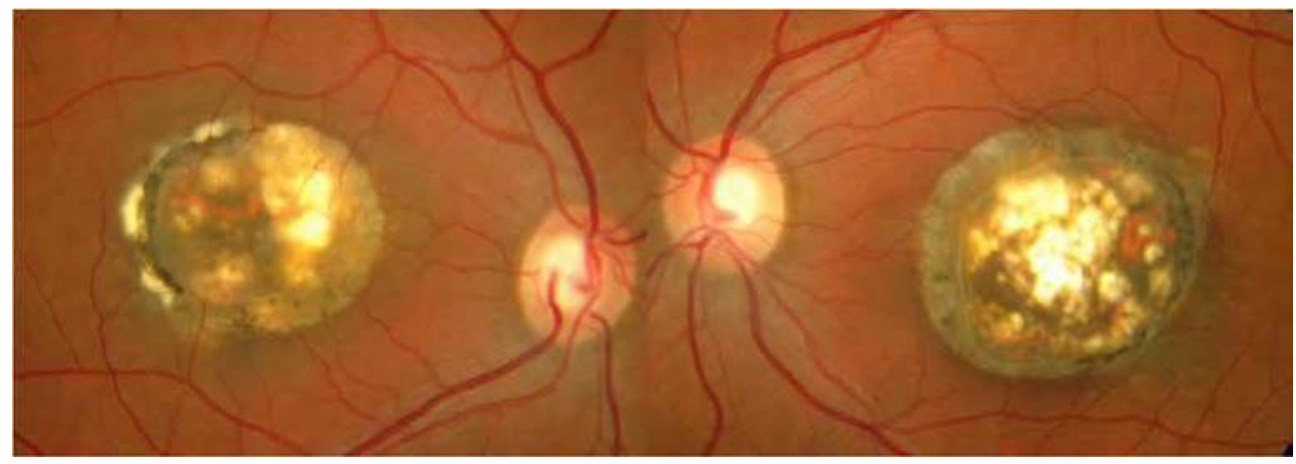

Fig. 37. A 41-year-old woman with a visual acuity of 5/10 in right eye and 3/10 in left eye. The reduced visual acuity is stable, and was already noted at the age of 12 years. There is a family history of maculopathy: the mother, the daughter and the brother of the patient are affected. In the fundus, the lesion is very large and extends over more than 6PD at the posterior pole, delimited in part by a fibrous band; the development of fibrosis can also be seen in the lesion.

The macular lesion characteristic of North Carolina dystrophy does not correspond to a coloboma or a staphyloma (Khurana et al, 2009). The term "crater" or "macular caldera" is therefore used. In this "crater" with steep edges, retinal tissue is limited to the innermost layers of the retina (fig 38). The lines representing the pigment epithelium and photoreceptors only reappear outside the lesion. 

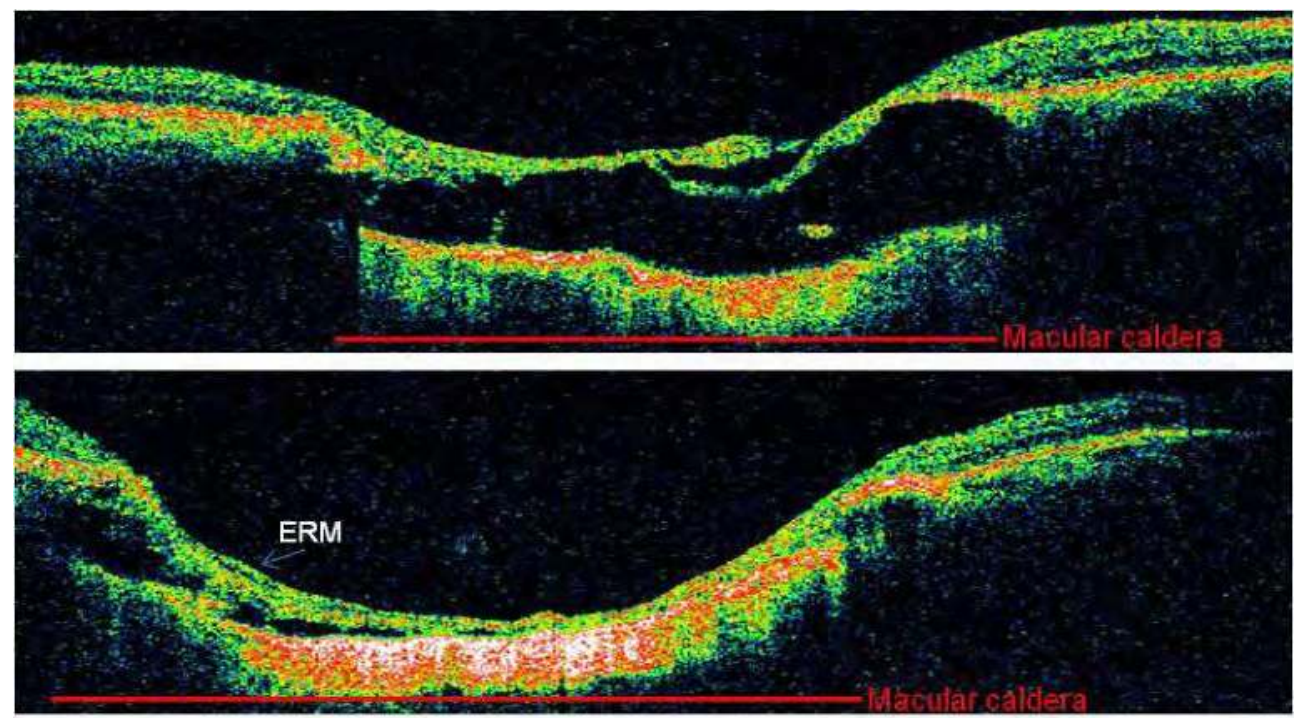

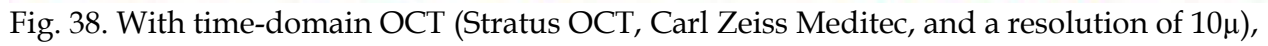
the lesion has the appearance of a crater with steep edges.

\section{Conclusion}

The possibility of visualizing in vivo all the retinal layers of the posterior pole, the different types of intraretinal and subretinal deposits and even choroidal abnormalities, using spectral-domain optical coherence tomography in combination with autofluorescence imaging, could lead to accurate diagnosis and phenotyping. These two imaging techniques have indisputably replaced fluorescein angiography in the study of inherited retinal dystrophies. OCT is very pertinent in revealing alterations to the neurosensory retina from very early stages, before any modification of the macula in the fundus. Nevertheless, the evaluation of disease state, disease progression and follow-up treatment are not as accurate beyond a certain degree of disorganization of the neurosensory retina.

\section{References}

Berger, W., Gruissem, BK., Neidhardt, J. The molecular basis of human retinal and vitreoretinal diseases. Prog Retin Eye Research, 2010, 29, pp. 335-375.

Ergun E, Hermann, B., \& Wirtitsch, M. Assessment of central visual function in Stargardt's disease/fundus flavimaculatus with ultrahigh-resolution optical coherence tomography. Invest Ophthalmol Vis Sci, 2005, 46, pp. 310-316.

Ferrara, DC., Costa, RA., \& Tsang, S. Multimodal fundus imaging in Best vitelliform macular dystrophy. Graefes Arch Clin Exp Ophthalmol, 2010, 248, pp. 1377-1386.

Gerth, C., Zawadzki, RJ., \& Werner, JS. Retinal microstructure in patients with EFEMP1 retinal dystrophy evaluated by Fourier domain OCT. Eye, 2009, 23, pp. 480-483. 
Hyewon, C., Jong-Uk, H., \& June-Gone, K. Optical coherence tomography in the diagnosis and monitoring of cystoids macular edema in patients with retinitis pigmentosa. Retina, 2006, 26, pp. 922-927.

Khurana, RH., Sun, X., Pearson, E. A reappraisal of the clinical spectrum of North Carolina dystrophy. Ophthalmology 2009, 116, pp. 1976-1983.

Querques, G., Leveziel, N., \& Benhamou, N. Analysis of retinal flecks in fundus flavimaculatus using optical coherence tomography. Br J Ophthalmol, 2006, 90, pp. 1157-1162.

Saihan, Z., Li, Z., \& Rice, J. Clinical and biochemical effects of the E139K missense mutation in the TIMP3 gene, associated with Sorsby fundus dystrophy. Mol Vis, 2009, 15, pp. 1218-1230.

Sandberg, MA., Brockhurst, RJ., \& Gaudio, AR. Visual acuity is related to parafoveal retinal thickness in patients with retinitis pigmentosa and macular cysts. Invest Ophthalmol Vis Sci, 2008, 49, pp. 4568-4572.

$\mathrm{Yu}, \mathrm{J} ., \mathrm{Ni}, \mathrm{Y} ., \quad \&$ Keane, PA. Foveomacular schisis in juvenile X-linked retinoschisis: an optical coherence tomography study. Am J Ophthalmol, 2010, 149, pp.973-978. 


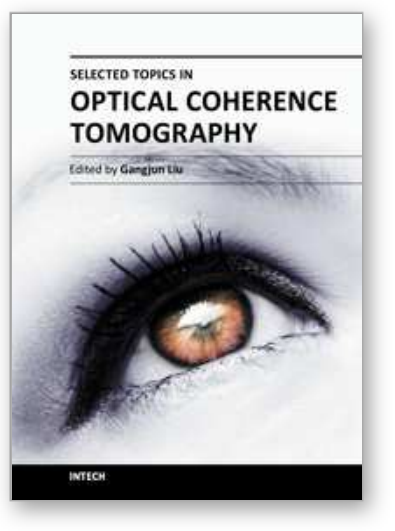

\author{
Selected Topics in Optical Coherence Tomography \\ Edited by Dr. Gangjun Liu
}

ISBN 978-953-51-0034-8

Hard cover, 280 pages

Publisher InTech

Published online 08, February, 2012

Published in print edition February, 2012

This book includes different exciting topics in the OCT fields, written by experts from all over the world. Technological developments, as well as clinical and industrial applications are covered. Some interesting topics like the ultrahigh resolution OCT, the functional extension of OCT and the full field OCT are reviewed, and the applications of OCT in ophthalmology, cardiology and dentistry are also addressed. I believe that a broad range of readers, such as students, researchers and physicians will benefit from this book.

\title{
How to reference
}

In order to correctly reference this scholarly work, feel free to copy and paste the following:

Isabelle Meunier, Carl Arndt, Xavier Zanlonghi, Sabine Defoort-Dhellemmes, Isabelle Drumare, Martine Mauget-Faysse, Benjamin Wolff, Aude Affortit, Christian Hamel and Bernard Puech (2012). Spectral-Domain Optical Coherence Tomography in Hereditary Retinal Dystrophies, Selected Topics in Optical Coherence Tomography, Dr. Gangjun Liu (Ed.), ISBN: 978-953-51-0034-8, InTech, Available from: http://www.intechopen.com/books/selected-topics-in-optical-coherence-tomography/spectral-domain-opticalcoherence-tomography-in-hereditary-retinal-dystrophies

\section{INTECH}

open science | open minds

\section{InTech Europe}

University Campus STeP Ri Slavka Krautzeka 83/A 51000 Rijeka, Croatia Phone: +385 (51) 770447 Fax: +385 (51) 686166 www.intechopen.com

\section{InTech China}

Unit 405, Office Block, Hotel Equatorial Shanghai No.65, Yan An Road (West), Shanghai, 200040, China 中国上海市延安西路65号上海国际贵都大饭店办公楼 405 单元 Phone: +86-21-62489820

Fax: $+86-21-62489821$ 
(C) 2012 The Author(s). Licensee IntechOpen. This is an open access article distributed under the terms of the Creative Commons Attribution 3.0 License, which permits unrestricted use, distribution, and reproduction in any medium, provided the original work is properly cited. 